\begin{tabular}{|c|l|}
\hline Title & Systematic investigation of the Hoyle anal og states in light nuclei \\
\hline Author(s) & Vasilevsky, V. S.; Kato, K.; Takibay ev, N. \\
\hline Citation & $\begin{array}{l}\text { Physical Review C, 98(2), 024325 } \\
\text { https://doi.org/10.1103/PhysRevC.98.024325 }\end{array}$ \\
\hline Issue Date & 2018.08.31 \\
\hline Doc URL & http://hdl.handle.net/2115/71837 \\
\hline Rights & @2018A American Physical Society \\
\hline Type & article \\
\hline File Information & PhysRevC.98.024325.pdf \\
\hline
\end{tabular}

Instructions for use 


\title{
Systematic investigation of the Hoyle-analog states in light nuclei
}

\author{
V. S. Vasilevsky* \\ Bogolyubov Institute for Theoretical Physics, Kiev 03143, Ukraine \\ K. Katō̄ \\ Nuclear Reaction Data Centre, Faculty of Science, Hokkaido University, Sapporo 060-0810, Japan \\ N. Takibayev \\ Al-Farabi Kazakh National University, Almaty 050040, Kazakhstan
}

(Received 9 June 2018; revised manuscript received 26 July 2018; published 31 August 2018)

\begin{abstract}
We investigate resonance states in three-cluster continuum of some light nuclei, ${ }^{9} \mathrm{Be},{ }^{9} \mathrm{~B},{ }^{10} \mathrm{~B},{ }^{11} \mathrm{~B}$, and ${ }^{11} \mathrm{C}$. These nuclei are considered to have a three-cluster configuration consisting of two $\alpha$ particles and a neutron, proton, deuteron, triton, and nucleus ${ }^{3} \mathrm{He}$, respectively. In this study, we make use of two different microscopic three-cluster models. The first model employs the hyperspherical harmonics basis to enumerate channels and describe three-cluster continuum. The second model is the well-known complex scaling method. The nucleonnucleon interaction is modeled by the semirealistic Minnesota and Hasegawa-Nagata potentials. Our main aim is to find the Hoyle-analog states in these nuclei or, in other words, whether it is possible to synthesize these nuclei in a triple collision of clusters. We formulate the criteria for selecting such states and apply them to resonance states, emerged from our calculations. We found that there are resonance states obeying the formulated criteria which make possible syntheses of these nuclei in a stellar environment.
\end{abstract}

DOI: 10.1103/PhysRevC.98.024325

\section{INTRODUCTION}

We are going to search for and analyze properties of the Hoyle-like states in light nuclei. It is necessary to recall that the Hoyle state is a very narrow resonance state in ${ }^{12} \mathrm{C}$, which was predicted by Fred Hoyle in 1954 [1]. Three years later this state was experimentally observed by studying $\beta$ decays of ${ }^{12} \mathrm{~B}$ [2]. It is interesting to note that Hoyle predicted the energy of the $0^{+}$resonance state at $E=0.33 \mathrm{MeV}$ above the three $\alpha$ particles threshold, and Cook et al. in Ref. [2] determined the position of the resonance state at $E=0.372 \pm 0.002 \mathrm{MeV}$. Compare this to the modern value of the energy, which is $E=$ $0.3796 \pm 0.0002 \mathrm{MeV}$ [3]. This resonance state, created by a triple collision of three $\alpha$ particles, is the key element in syntheses of atomic nuclei starting from ${ }^{12} \mathrm{C}$. The Hoyle state indicates a way for the nucleosynthesis of carbon in heliumburning red giant stars, which are rich in $\alpha$ particles. Actually, Hoyle was the first to proclaim that nuclear synthesis can take place in a triple collision of light nuclei, namely $\alpha$ particles. Such processes are very difficult to acheive in the laboratory, but natural conditions inside stars enable such processes. One can find more interesting historical facts and scientific results about the Hoyle state in the review [4].

We consider two important quotations from the Hoyle paper [1]:

\footnotetext{
*vsvasilevsky@gmail.com

${ }^{\dagger}$ kato@nucl.sci.hokudai.ac.jp

†takibayev@gmail.com
}

(1) "It was pointed out some years ago by H. Bethe [5] that effective element-building inside starts must proceed, in the absence of hydrogen, by triple collisions as a starting point:

$$
3 \alpha \rightarrow{ }^{12} \mathrm{C}+\gamma . "
$$

(2) "It is convenient to replace reaction (1) by

$$
\alpha+\alpha \rightarrow{ }^{8} \mathrm{Be}, \quad{ }^{8} \mathrm{Be}+\alpha \rightarrow{ }^{12} \mathrm{C}+\gamma .
$$

This is a permissible step, since the lifetime of the unstable ${ }^{8} \mathrm{Be}$ is appreciably longer than the time required for nuclear collision of two $\alpha$ particles; that is, longer than the $\alpha$ particle radius divided by the relative velocity."

These two equations (1) and (2) represent two different ways of excitation of the Hoyle resonance state and two different ways of synthesis of ${ }^{12} \mathrm{C}$. However, in both scenarios the very narrow $0^{+}$resonance state is the key factor in creation of the carbon-12.

There are a very large number of publications devoted to the $0^{+}$and other resonance states in ${ }^{12} \mathrm{C}$. Different methods have been used to determine parameters of the Hoyle state and to shed some light on the nature of this states and other resonances states, residing in the three-cluster continuum in ${ }^{12} \mathrm{C}$. However, only a few publications [6-13] have been aimed at finding the Hoyle-analog states in light nuclei. They are mainly concentrated on the closest neighbors of the ${ }^{12} \mathrm{C}$ nucleus, namely ${ }^{11} \mathrm{~B},{ }^{11} \mathrm{C}$, and ${ }^{13} \mathrm{C}$. In Refs. [7] and [10] the structure of $1 / 2^{+}$and $3 / 2^{-}$states in ${ }^{11} \mathrm{~B}$ has been investigated within the three-cluster orthogonality condition 
model (OCM) combined with the Gauss expansion method. In these papers, parameters of resonance states were obtained by using the complex scaling technique. By analyzing properties of wave functions, the authors of the Refs. $[7,10]$ came to the conclusion that for the $1 / 2^{+}$resonance state the parameters $E=0.75 \mathrm{MeV}$ and $\Gamma=190 \mathrm{keV}$ can be considered as the Hoyle-analog states. Antisymmetrized molecular dynamics (AMD) was used to study the excited states of the negative parity in ${ }^{11} \mathrm{~B}$ and ${ }^{11} \mathrm{C}$ in Refs. [6,8]. It was concluded that the third excited states in ${ }^{11} \mathrm{~B}$ and ${ }^{11} \mathrm{C}$ have dilute cluster structures $\alpha+\alpha+t$ and $\alpha+\alpha+{ }^{3} \mathrm{He}$, respectively, and can be treated as the Hoyle-analog states.

In the present paper we consider these nuclei and also ${ }^{9} \mathrm{Be}$, ${ }^{9} \mathrm{~B}$, and ${ }^{10} \mathrm{~B}$. We also consider a large number of states with different values of the total momentum $J$ and both negative and positive parities. Before starting to search for the Hoyle-analog states, one needs to formulate clear criteria for selecting such states. By analyzing properties of the Hoyle state, one may suggest the following criteria for the Hoyle-analog states in three-cluster systems:

(1) very narrow resonance state;

(2) resonance state which lies close to three-cluster threshold;

(3) resonance state which has the total orbital momentum $L=0$.

We consider the first criterion as the most important because, in the case of very narrow (long-lived) resonance states, a compound system has more chances to be reconstructed and transformed in to a bound state. However, we will analyze all resonance states from the point of view of the three criteria.

Our main aim is to find the Hoyle-analog states in light nuclei ${ }^{9} \mathrm{Be},{ }^{9} \mathrm{~B},{ }^{10} \mathrm{~B},{ }^{11} \mathrm{~B}$ and ${ }^{11} \mathrm{C}$. In other words, we are going to study whether light nuclei can be created in triple collision of clusters. The necessary condition for such a process is the existence of a very narrow resonance state in the three-cluster continuum. Actually we consider a chain of reactions

$$
A_{1}+A_{2}+A_{3}=A^{*} \Rightarrow A+\gamma,
$$

which consists of two steps. In the first step, an excited state (very narrow resonance state) of a compound nucleus is created in a triple collision of clusters consisting of $A_{1}, A_{2}$, and $A_{3}$ nucleons. In the second step, the compound nucleus, by emitting a photon, transitions from the resonance state to the bound state. The narrower a resonance state is in the first step, the higher the probability is of transitioning from the resonance to the bound state. For each nucleus we determine energy and width of resonance states. We select a resonance state with a very small width. We also analyze the wave function of selected resonance states. These investigations will be performed within a microscopic three-cluster model which involves hyperspherical harmonics to distinguish channels of the three-cluster system. For this model, which was formulated in Ref. [14], we use the abbreviation AMHHB, which means the algebraic model of scattering making use of the hyperspherical harmonics basis. In Ref. [15] this model was applied to study bound and resonance states in ${ }^{12} \mathrm{C}$. It fairly well reproduced the energy and width of the Hoyle state in ${ }^{12} \mathrm{C}$. It was demonstrated that this model is also in good agreement with other alternative models, for instance, the complex scaling method. Note that the most effective methods, among the others that are used to study resonance states in three-cluster and many-channel systems, are the complex scaling method and hyperspherical harmonics method

We present results obtained with both methods. The AMHHB method, which employs hyperspherical harmonics to enumerate channels of three-cluster continuum, allows us to determine energy and width of a resonance state, reveals the dominant decay channels, and sheds more light on the nature of the resonance state by analyzing its wave functions. This model correctly treats the Pauli principle and makes uses of the semi-realistic nucleon-nucleon potential. The complex scaling method (CSM), which also uses this type of the nucleon-nucleon interaction, is more a advanced and model independent method to determine the poles of the $S$ matrix in two- and three-cluster systems. Note that both methods give very close results for narrow resonance states and different resonance parameters for wide resonance states.

The preliminary analysis of three-cluster resonance states in ${ }^{9} \mathrm{~B}$ and ${ }^{9} \mathrm{~B}$ was carried out in Ref. [16], and resonance states were investigated in the mirror nuclei ${ }^{11} \mathrm{~B}$ and ${ }^{11} \mathrm{C}$ in Ref. [17]. In Ref. [18] the AMHHB model was applied to study the spectrum of bound states in ${ }^{10} \mathrm{~B}$. To make a systematic analysis of resonance states and to discover the Hoyle-analog states in ${ }^{9} \mathrm{Be},{ }^{9} \mathrm{~B},{ }^{10} \mathrm{~B},{ }^{11} \mathrm{~B}$, and ${ }^{11} \mathrm{C}$ we have to make additional calculations and thorough investigations of peculiarities of resonance wave functions.

The present paper is organized in the following way. In Sec. II we briefly explain the main idea of the microscopic method, which involves hyperspherical harmonics for description of bound and scattering states of a three-cluster system. Results of numerical calculations and discussions of the results obtained are presented in Sec. III. We start with the reexamination of properties of the Hoyle state. We also consider other resonance states in ${ }^{12} \mathrm{C}$ to display similarities and differences between them. This is done within the AMHHB and CSM in order to formulate clearer criteria for selecting the Hoyle-analog states. Then we proceed with analysis of resonance states in the three-cluster continuum of nuclei ${ }^{9} \mathrm{Be}$, ${ }^{9} \mathrm{~B},{ }^{10} \mathrm{~B},{ }^{11} \mathrm{~B}$, and ${ }^{11} \mathrm{C}$. By applying the formulated criteria, we select the Hoyle-analog states and describe their properties. Section IV presents a summary of our investigations.

\section{METHOD}

\section{A. Three-cluster wave function}

To study three-cluster systems we exploit a microscopic model which incorporates the resonating group method, the $J$ matrix method, or the algebraic version of the resonating group method (RGM) and the hyperspherical harmonics method. Details of the model and its application to the study of bound and continuous spectrum states of light nuclei can be found in Refs. [14,15,17,19-22].

The standard ansatz of the RGM for representing the wave function of a three- $s$-cluster system is used:

$$
\Psi_{E, J}=\sum_{S, L} \widehat{\mathcal{A}}\left\{\left[\Phi_{1}\left(A_{1}\right) \Phi_{2}\left(A_{2}\right) \Phi_{3}\left(A_{3}\right)\right]_{S} \psi_{E, \mathrm{LJ}}(\mathbf{x}, \mathbf{y})\right\}_{J}
$$


where the wave function $\psi_{E, \mathrm{LJ}}(\mathbf{x}, \mathbf{y})$ describes relative motion of clusters and the antisymmetric functions $\Phi_{\nu}\left(A_{\nu}\right)(v=$ $1,2,3)$ describe internal motion of nucleons inside the cluster with index $v$. Two vectors $\mathbf{x}$ and $\mathbf{y}$ denote a possible set of the Jacobi vectors. Within this paper, the vector $\mathbf{x}$ determines the distance between two selected clusters, while the vector $\mathbf{y}$ represents displacement of the third cluster with respect to the center of mass of the two selected clusters. The antisymmetrization operator $\widehat{\mathcal{A}}$ provides full antisymmetrization of the wave function of a compound system. By assuming $\widehat{\mathcal{A}}=1$ and the orthogonality condition to the Paul-forbidden states, one transitions to the OCM.

It is very convenient to use the $L S$ coupling scheme for three interacting $s$ clusters. In this scheme, the total spin $S$ is a vector sum of individual spins of clusters, and the total orbital momentum $L$ is also a vector sum of the partial orbital momenta $\widehat{\mathbf{l}}_{x}$ and $\widehat{\mathbf{l}}_{y}$, associated with the Jacobi vectors $\mathbf{x}$ and $\mathbf{y}$, respectively. The total angular momentum $J$ is a vector sum of the total orbital momentum $L$ and the total spin $S$.

To simplify obtaining wave functions of discrete and continuous spectrum states and scattering parameters, we transform from the Jacobi vectors $\mathbf{x}$ and $\mathbf{y}$ to the hyperspherical coordinates, which consist of hyperradius $\rho$ and five hyperspherical angles which we denote as $\Omega_{5}$. The hyperradius $\rho$ is defined in the unambiguous way

$$
\rho=\sqrt{\mathbf{x}^{2}+\mathbf{y}^{2}}
$$

while there are several different ways to define of the hyperspherical angles (see, for instance, [23-25]). We make use of the most popular set of hyperspherical angles, which was suggested by Zernike and Brinkman in 1935 [26]. This set consists of the hyperspherical angle $\theta$ which determines relative lengths of the Jacobi vectors

$$
x=\rho \cos \theta, \quad y=\rho \sin \theta,
$$

two angles $\theta_{x}$ and $\phi_{x}$, determining orientation of vector $\mathbf{x}$, and two other angles $\theta_{y}$ and $\phi_{y}$, determining orientation of vector $\mathbf{y}$ in the space. Note, that the angles $\left\{\theta_{x}, \phi_{x}\right\}$ describe rotation of a two-cluster subsystem and the angles $\left\{\theta_{y}, \phi_{y}\right\}$ describe rotation of the third cluster around center of mass of the two-cluster subsystem. Five hyperspherical angles are able to describe any shape and any orientation (i.e., rotation) of a triangle connecting centers of mass of three clusters, and the hyperradius determines any size of that triangle.

Having introduced the hyperspherical coordinate, we can represent the three-cluster wave function (3) in the following form:

$$
\begin{aligned}
\Psi_{E, J}= & \sum_{c} \widehat{\mathcal{A}}\left\{\left[\Phi_{1}\left(A_{1}\right) \Phi_{2}\left(A_{2}\right) \Phi_{3}\left(A_{3}\right)\right]_{S}\right. \\
& \left.\times \psi_{E, c}(\rho) \mathcal{Y}_{c}\left(\Omega_{5}\right)\right\}_{J},
\end{aligned}
$$

where $c$ is a multiple index $c=\{K ; \lambda, l ; L, S\}$ classifying channels of the three-cluster system and involving the hypermomentum $K$, partial orbital momenta $\lambda$ and $l$ associated with the Jacobi vectors $\mathbf{x}$ and $\mathbf{y}$, respectively, and the total orbital momentum $L$. The hyperspherical harmonics $\mathcal{Y}_{c}\left(\Omega_{5}\right)$ form a complete set of functions on a five-dimension sphere and thus account for all kinds of motion of a three-cluster system.
Components of the many-channel hyperradial wave function $\left\{\psi_{E, c}(\rho)\right\}$ have to be determined by solving the Schrödinger equation with the selected nucleon-nucleon potential.

\section{B. Three-cluster equation}

For three structureless particles one obtains the infinite set of differential equations

$$
\sum_{\widetilde{c}}\left[\delta_{c, \widetilde{c}} \widehat{T}_{K}+V_{c, \widetilde{c}}(\rho)\right] \psi_{E, \widetilde{c}}(\rho)=E \psi_{E, c}(\rho),
$$

where

$$
\widehat{T}_{K}=-\frac{\hbar^{2}}{2 m}\left[\frac{\partial^{2}}{\partial \rho^{2}}+\frac{5}{\rho} \frac{\partial}{\partial \rho}-\frac{K(K+4)}{\rho^{2}}\right] .
$$

Matrix $\left\|V_{c, \widetilde{c}}(\rho)\right\|$ of the effective potential energy is determined as matrix elements of interaction $\widehat{V}$ between the hyperspherical harmonics

$$
V_{c, \widetilde{c}}(\rho)=\left\langle\mathcal{Y}_{c}|\widehat{V}| \mathcal{Y}_{\widetilde{c}}\right\rangle,
$$

where integration is performed over all hyperspherical angles $\Omega_{5}$. If particles have electric charges, then we have the contribution

$$
V_{c, \widetilde{c}}^{(C)}(\rho)=\frac{Z_{c, \widetilde{c}} e^{2}}{\rho}
$$

from the Coulomb interaction to the potential energy $V_{c, \widetilde{c}}(\rho)$ (9). The quantity $Z_{c, \tilde{c}}$ can be called the effective charge. Assuming that at a large values of hyperradius the effective potential $V_{c, \widetilde{c}}(\rho)$ originating from a short range particle-particle interaction is negligibly small, and omitting nondiagonal elements of the effective charge (that is putting $Z_{c, \widetilde{c}}=0$ for $c \neq \widetilde{c}$ ), we obtain an asymptotic part of the channel Hamiltonian,

$$
\widehat{H}_{c}^{(A)}=\left\{-\frac{\hbar^{2}}{2 m}\left[\frac{\partial^{2}}{\partial \rho^{2}}+\frac{5}{\rho} \frac{\partial}{\partial \rho}-\frac{K(K+4)}{\rho^{2}}\right]+\frac{Z_{c, c} e^{2}}{\rho}\right\} .
$$

Eigenfunctions of this Hamiltonian describing incoming and outgoing hyperradial waves can be easily found and expressed through the Whittaker functions (see Chap. 13.1 in Ref. [27])

$$
\psi_{c}^{( \pm)}\left(\rho, \eta_{c}\right)=\sqrt{\frac{\pi}{2}} \frac{1}{\rho^{5 / 2}} W_{\mp i \eta_{c}, K+2}(\mp 2 i k \rho),
$$

where

$$
k=\sqrt{\frac{2 m E}{\hbar^{2}}}
$$

and $\eta_{c}$ is the Sommerfeld parameter for the three-cluster system,

$$
\eta_{c}=\frac{m}{\hbar^{2}} \frac{Z_{c, c} e^{2}}{k} .
$$

Thus, the boundary conditions or the asymptotic form of manychannel wave functions can be expressed in the form

$$
\psi_{E, c}(\rho)=\delta_{c_{0}, c} \psi_{c}^{(-)}\left(\rho, \eta_{c}\right)-S_{c_{0}, c} \psi_{c}^{(+)}\left(\rho, \eta_{c}\right),
$$

where $c_{0}$ stands for an incoming channel and $S_{c_{0}, c}$ is an element of the scattering $S$ matrix. 
For three-cluster systems, when the internal structure of clusters and the Pauli principle are taken into account, we obtain the set of integrodifferential equations

$$
\begin{aligned}
& \sum_{\widetilde{c}}\left[\delta_{c, \widetilde{c}} \widehat{T}_{K} \psi_{E, \widetilde{c}}(\rho)+\int d \widetilde{\rho} \widetilde{\rho}^{5} V_{c, \widetilde{c}}(\rho, \widetilde{\rho}) \psi_{E, \widetilde{c}}(\widetilde{\rho})\right] \\
& \quad=E \sum_{\widetilde{c}} \int d \widetilde{\rho} \widetilde{\rho}^{5} N_{c, \widetilde{c}}(\rho, \widetilde{\rho}) \psi_{E, \widetilde{c}}(\widetilde{\rho}) .
\end{aligned}
$$

This system of equations can be obtained from the manyparticle Schrödinger equations with the help of the projection operator

$$
\widehat{P}_{c}(\rho)=\widehat{\mathcal{A}}\left\{\left[\Phi_{1}\left(A_{1}\right) \Phi_{2}\left(A_{2}\right) \Phi_{3}\left(A_{3}\right)\right]_{S} \delta(\rho-\bar{\rho}) \mathcal{Y}_{c}\left(\Omega_{5}\right)\right\} .
$$

Applying this operator to the unit operator, we obtain the norm kernel $N_{c, \widetilde{c}}(\rho, \widetilde{\rho})$ :

$$
N_{c, \widetilde{c}}(\rho, \widetilde{\rho})=\left\langle\widehat{P}_{c}(\rho) \mid \widehat{P}_{\widetilde{c}}(\widetilde{\rho})\right\rangle .
$$

In this expression integration is performed over all spacial coordinates (the Jacobi vectors) and over all spin and isospin coordinates as well. The matrix of the potential energy is related to matrix elements of the microscopic Hamiltonian $\widehat{H}$ by the relation

$$
V_{c, \widetilde{c}}(\rho, \widetilde{\rho})=\left\langle\widehat{P}_{c}(\rho)|\widehat{H}| \widehat{P}_{\widetilde{c}}(\widetilde{\rho})\right\rangle-\delta_{c, \widetilde{c}} \widehat{T}_{K} \delta(\rho-\widetilde{\rho}) .
$$

The system of Equations (13) can be directly solved by reducing to the reasonable finite number of involved three-cluster channels $N_{c}$ and with the boundary conditions determined above. Solutions of the system yield the definite set of matrix elements of the $S$ matrix. They describe all kinds of elastic and inelastic processes in a three-cluster system.

Note that the operator (14) is a straightforward generation of the projection operator that has been used for two-cluster systems (see Ref. [28]). In three-cluster systems, we can easily perform this operation, though we do not explain the details here.

Within the present model a wave function (3) of a threecluster system is expanded over an infinite set of cluster oscillator functions $\left|n_{\rho}, c\right\rangle$,

$$
\Psi_{E, \mathrm{LJ}}=\sum_{n_{\rho}, c} C_{n_{\rho}, c}^{E, J}\left|n_{\rho}, c\right\rangle,
$$

where

$$
\begin{aligned}
\left|n_{\rho}, c\right\rangle & =\left|n_{\rho}, K ; \lambda, l ; L\right\rangle \\
& =\widehat{\mathcal{A}}\left\{\Phi_{1}\left(A_{1}\right) \Phi_{2}\left(A_{2}\right) \Phi_{3}\left(A_{3}\right) R_{n_{\rho} K}(\rho, b) \mathcal{Y}_{c}\left(\Omega_{5}\right)\right\}
\end{aligned}
$$

$\mathcal{Y}_{c}\left(\Omega_{5}\right)$ is a hyperspherical harmonic with the quantum numbers $c=\left\{K, l_{x}, l_{y}, L\right\}, R_{n_{\rho}, K}(\rho, b)$ is an oscillator function,

$$
\begin{array}{r}
R_{n_{\rho}, K}(\rho, b)=(-1)^{n_{\rho}} \mathcal{N}_{n_{\rho}, K} r^{K} \exp \left\{-\frac{1}{2} r^{2}\right\} L_{n_{\rho}}^{K+3}\left(r^{2}\right), \\
r=\rho / b, \quad \mathcal{N}_{n_{\rho}, K}=b^{-3} \sqrt{\frac{2 \Gamma\left(n_{\rho}+1\right)}{\Gamma\left(n_{\rho}+K+3\right)}},
\end{array}
$$

and $b$ is an oscillator length.
In this case, a set of the integro-differential equations is reduced to a set of the algebraic (matrix) equations,

$$
\sum_{\widetilde{n}_{\rho}, \widetilde{c}}\left[\left\langle n_{\rho}, c|\widehat{H}| \widetilde{n}_{\rho}, \widetilde{c}\right\rangle-E\left\langle n_{\rho}, c \mid \widetilde{n}_{\rho}, \widetilde{c}\right\rangle\right] C_{\widetilde{n}_{\rho}, \widetilde{c}}^{E, J}=0,
$$

which can be more easily solved by the numerical methods than the set of Eq. (13). For continuous spectrum states one has to impose proper boundary conditions for expansion coefficients $\left\{C_{n_{\rho}, c}^{E, J}\right\}$. These conditions have been discussed in Ref. [14], where relations between the discrete $\left\{C_{n_{\rho}, c}^{E, J}\right\}$ and continuous $\left\{\psi_{E, c}(\rho)\right\}$ wave functions were established. By including the asymptotic form of expansion coefficients $\left\{C_{n_{\rho}, c}^{E, J}\right\}$, which is valid for large values of hyperradial excitations $n_{\rho} \gg 1$, we obtain in a closed form the system of equations determining both wave functions of a continuous spectrum and the corresponding $S$ matrix.

\section{Supplementary quantities}

Having obtained the expansion coefficients for any state of the three-cluster continuum, we can easily construct its wave function in the coordinate space. It can be done, first of all, for the total hyperradial wave function

$$
\psi_{E, c}(\rho)=\sum_{n_{\rho}} C_{n_{\rho}, c}^{E, J} R_{n_{\rho}, K}(\rho, b) .
$$

It can be also done for the wave function

$$
\psi_{E, \mathrm{LJ}}(\mathbf{x}, \mathbf{y})=\sum_{n_{\rho}, c} C_{n_{\rho}, c}^{E, J} R_{n_{\rho}, K}(\rho, b) \mathcal{Y}_{c}\left(\Omega_{5}\right) .
$$

To get more information about the state under consideration, we will study different quantities that can be obtained with the wave function in discrete or coordinate spaces. With wave functions in the discrete oscillator quantum number representation we can determine a weight $W_{\text {sh }}$ of the oscillator function belonging to the oscillator shell $N_{\mathrm{sh}}$ in this wave function:

$$
W_{\mathrm{sh}}\left(N_{\mathrm{sh}}\right)=\sum_{n_{\rho}, c \in N_{\mathrm{sh}}}\left|C_{n_{\rho}, c}^{E, J}\right|^{2}
$$

where the summation is performed over all hyperspherical harmonics and hyperradial excitations obeying the condition

$$
N_{\mathrm{os}}=2 n_{\rho}+K \text {. }
$$

Here $N_{\text {os }}$ is fixed. Basis wave functions (18) belong to the oscillator shell with the number of oscillator quanta $N_{\mathrm{os}}=$ $2 n_{\rho}+K$. It is convenient to enumerate the oscillator shells by $N_{\text {sh }}(=0,1,2, \ldots)$, which we determine as

$$
N_{\mathrm{os}}=2 n_{\rho}+K=2 N_{\mathrm{sh}}+K_{\mathrm{min}},
$$

where $K_{\min }=L$ for normal parity states $\pi=(-1)^{L}$ and $K_{\min }=L+1$ for abnormal parity states $\pi=(-1)^{L+1}$. Thus we account oscillator shells starting from a "vacuum" shell $\left(N_{\mathrm{sh}}=0\right)$ with the minimal value of the hypermomentum $K_{\text {min }}$ compatible with a given total orbital momentum $L$.

The weights $W_{\text {sh }}$ we will calculate both for bound and resonance states. For a bound state, the wave function is 
normalized by the condition

$$
\left\langle\Psi_{E, J} \mid \Psi_{E, J}\right\rangle=\sum_{n_{\rho}, c}\left|C_{n_{\rho}, c}^{E, J}\right|^{2}=1,
$$

and this quantity $W_{\text {sh }}$ determines the probability. For the continuous spectrum state, when the wave function is normalized by the condition

$$
\left\langle\Psi_{E, J} \mid \Psi_{\widetilde{E}, J}\right\rangle=\sum_{n_{\rho}, c} C_{n_{\rho}, c}^{E, J} C_{n_{\rho}, c}^{\widetilde{E}, J}=\delta(k-\widetilde{k}),
$$

this quantity has a different meaning. It determines the relative contribution of the different oscillator shells and also the shape of the resonance wave function in the oscillator representation.

It is worthwhile to notice that oscillator functions have some important features. Oscillator functions belonging to an oscillator shell $N_{\mathrm{sh}}$ allow one to describe a many-particle system in a finite range of hyperradius $0<\rho \leqslant b \sqrt{4 N_{\mathrm{sh}}+K_{\min }+3}$. Outside this region, these oscillator functions give a negligibly small contribution to the many-particle wave function. This statement is, for example, demonstrated in Ref. [29]. Thus, oscillator functions with a small value of $N_{\mathrm{sh}}$ describe very compact configurations of a three-cluster system with all clusters being close to each other. When $N_{\text {sh }}$ is large, the oscillator functions represent dispersed (dilute) configurations. There are two principal regimes in these configurations. The first regime is associated with a two-body type of asymptotic when two clusters are at a small distance and the third cluster is moved far away. The second regime accounts for the case when all three clusters are well separated. Taking these into account, we will deduce from an analysis of shell weights $W_{\text {sh }}$ whether a wave function of a bound or resonance state describes a compact or dispersed three-cluster configuration.

It is necessary to add another important remark. There is a strict correspondence between wave functions in the oscillator and coordinate spaces [30,31]. In Ref. [14] a correspondence was established between the expansion coefficients $\left\{C_{n_{\rho}, c}^{E, J}\right\}$ and coordinate wave function $\psi_{E, c}(\rho)$ for three-cluster systems. It reads as

$$
C_{n_{\rho}, c}^{E, J} \approx \sqrt{2} \rho_{n}^{2} \psi_{E, c}\left(\rho_{n}\right)
$$

where

$$
\rho_{n}=b \sqrt{4 n_{\rho}+2 K+3}
$$

is a coordinate of the classical turning point in the sixdimension harmonic oscillator. Formally, this correspondence is valid for a large values of $n_{\rho}$; however, in real cases this correspondence is also valid for moderate values of $n_{\rho}$. One may deduce from this correspondence that the weights $W_{\mathrm{sh}}\left(N_{\mathrm{sh}}\right)$ for large values of $N_{\mathrm{sh}}$ are proportional to the squared modulus of the wave function $\Psi_{E, J}$ [Eq. (3)] at a discrete value of hyperradius $\rho=b \sqrt{4 N_{\mathrm{sh}}+2 K_{\min }+3}$. From the other side, the correspondence (25) confirms the above statement that the weights $W_{\mathrm{sh}}\left(N_{\mathrm{sh}}\right)$ with a small values of $N_{\mathrm{sh}}$ describe a compact configuration of a three-cluster system, while the weights $W_{\text {sh }}\left(N_{\text {sh }}\right)$ for a large values of $N_{\text {sh }}$ determine the probability of a dispersed configuration of the three-cluster system.
By employing the wave function in the coordinate space, we determine the correlation function

$$
D(x, y)=x^{2} y^{2} \int\left|\psi_{E, \mathrm{LJ}}(\mathbf{x}, \mathbf{y})\right|^{2} d \widehat{\mathbf{x}} d \widehat{\mathbf{y}}
$$

and average distances $R_{1}$ and $R_{2}$ between clusters,

$$
\begin{array}{r}
R_{1}=\sqrt{\frac{A}{\left(A_{1}+A_{2}\right) A_{3}}} \sqrt{\int y^{2}\left|\psi_{E, \mathrm{LJ}}(\mathbf{x}, \mathbf{y})\right|^{2} d \mathbf{x} d \mathbf{y}}, \\
R_{2}=\sqrt{\frac{\left(A_{1}+A_{2}\right)}{A_{1} A_{2}}} \sqrt{\int x^{2}\left|\psi_{E, \mathrm{LJ}}(\mathbf{x}, \mathbf{y})\right|^{2} d \mathbf{x} d \mathbf{y}} .
\end{array}
$$

In our notations, $R_{2}$ determines an average distance between $\alpha$ particles, while $R_{1}$ determines a distance of the third cluster to the center of mass of two $\alpha$ particles. Note that in Eq. (26) integration is performed over unit vectors $\widehat{\mathbf{x}}$ and $\widehat{\mathbf{y}}$, while in Eqs. (27) and (28) integration is carried out over all Jacobi vectors or all hyperspherical coordinates.

It is obvious that the correlation function $D(x, y)$ can be determined both for bound and resonance states. However, the average distances $R_{1}$ and $R_{2}$ can be calculated for the bound state only, since for resonance states the integrals in Eqs. (27) and (28) diverge. In Ref. [16] we suggested to extend to resonance states the definition of average distances $R_{1}$ and $R_{2}$. For this aim we restricted the integration within the internal part of the resonance wave functions, which was normalized to unity. Recall that the internal part of a wave function is represented in the region $\left(0 \leqslant \rho \leqslant \rho_{\max }\right.$ in the coordinate space or $0 \leqslant n_{\rho} \leqslant N^{(i)}$ in the oscillator space) where distances between clusters are relatively small and effects intercluster interactions are very strong. Such a definition of $R_{1}$ and $R_{2}$ allows us to study the shape of the triangle composed by three interacting clusters, but not its size. By comparing average distances $R_{1}$ and $R_{2}$ for different resonances of the same or other nucleus, we obtain more information on the structure of the resonance wave functions.

It is important to note that the oscillator basis (17) can be used to determine parameters of resonance states within the methodology of the complex scaling method. This will be demonstrated in another paper. However, it is more expedient to use the Gaussian basis in the six-dimension space to perform this type of calculations, as this basis provides more rapid convergence of results than the oscillator basis.

\section{RESULTS AND DISCUSSIONS}

For all nuclei under consideration we employ the Minnesota potential [32,33] (MP) or the modified Hasegawa-Nagata potential [34,35] (MHNP). Both the central and spin-orbital components of these potentials are taken into account.

In such calculations we have only one free parameter to be selected. This is the oscillator length $b$ which is common for all clusters of a compound nucleus and effectively determines the spatial distribution of nucleons in clusters. In our calculations the oscillator length $b$ is fixed by minimizing the energy of the three-cluster threshold. For ${ }^{9} \mathrm{Be},{ }^{9} \mathrm{~B}$, and ${ }^{12} \mathrm{C}$, the oscillator length $b$ minimizes the energy of an $\alpha$ particle.

The Majorana parameter $m$ of the MHNP and the exchange parameter $u$ of the MP are very often used as an adjustable parameter. If one adjusts these parameters to reproduce phase 
TABLE I. List of nuclei investigated within microscopic threecluster compound (3CC) models and different input parameters; $b$ is the oscillator length, $m$ or $u$ are exchange parameters of the nucleonnucleon interaction.

\begin{tabular}{lcccc}
\hline \hline Nucleus & 3CC & Potential & $u$ or $m$ & $b(\mathrm{fm})$ \\
\hline${ }^{9} \mathrm{Be}$ & $\alpha+\alpha+n$ & MHNP & 0.0332 & 1.317 \\
${ }^{9} \mathrm{~B}$ & $\alpha+\alpha+p$ & MHNP & 0.0332 & 1.317 \\
${ }^{10} \mathrm{~B}$ & $\alpha+\alpha+d$ & MP & 0.915 & 1.395 \\
${ }^{11} \mathrm{~B}$ & $\alpha+\alpha+{ }^{3} \mathrm{H}$ & MP & 0.920 & 1.322 \\
${ }^{11} \mathrm{C}$ & $\alpha+\alpha+{ }^{3} \mathrm{He}$ & MP & 0.920 & 1.322 \\
${ }^{12} \mathrm{C}$ & $\alpha+\alpha+\alpha$ & MP & 0.940 & 1.285 \\
\hline \hline
\end{tabular}

shifts of the $\alpha-\alpha$ scattering and parameters of resonance states in ${ }^{8} \mathrm{Be}$, one obtains the overbound $0^{+}$and $2^{+}$states in ${ }^{12} \mathrm{C}$, and also the $4^{+}$bound state, which contradicts the experimental data. This problem was discussed in Ref. [15] where the MP was used to calculate the spectrum of bound and resonance states in ${ }^{12} \mathrm{C}$. Such a problem also appears for all nuclei under consideration. This problem has been discussed in Refs. [16,22], where spectra of ${ }^{9} \mathrm{~B}$ and ${ }^{9} \mathrm{Be}$ were investigated. To avoid the appearance unphysical bound states, we adjust parameters $m$ and $u$ to reproduce the energy of the ground state measured from the three-cluster threshold. For mirror nuclei ${ }^{9} \mathrm{Be},{ }^{9} \mathrm{~B}$ and ${ }^{11} \mathrm{~B},{ }^{11} \mathrm{C}$ we adjust these parameters only for one nucleus of these pairs; for ${ }^{9} \mathrm{~B}$ and ${ }^{11} \mathrm{~B}$. This is done in order to study effects of the Coulomb interaction on parameters of bound and resonance states. In Table I we collected input parameters for each nucleus.

In Table II we display energies of the ground state of two-cluster subsystems for all selected nuclei. These results are obtained with the input parameters indicated in Table I. For nuclei ${ }^{6} \mathrm{Li},{ }^{7} \mathrm{Li}$, and ${ }^{7} \mathrm{Be}$ we show the bound state energies, while for nuclei ${ }^{5} \mathrm{He},{ }^{5} \mathrm{Li}$, and ${ }^{8} \mathrm{Be}$ we present energies and widths of the resonance states, which are usually considered as the ground states. One can see that, by selecting the optimal values of the parameters $u$ and $m$ of the nucleon-nucleon interactions, we make the very broad $0^{+}$resonance state in ${ }^{8} \mathrm{Be}$. We can also see in Table II that the nucleon-nucleon interactions with the selected parameters give the overbound ground states of ${ }^{5} \mathrm{He}(0.348 \mathrm{MeV})$ and ${ }^{5} \mathrm{Li}(1.236 \mathrm{MeV})$ for the observed $3 / 2^{-}$ resonance energies 0.798 and $1.69 \mathrm{MeV}$, respectively. These

TABLE II. Ground states of the binary subsystems. Here 3CC stands for the three-cluster compound system and 2CC indicates two-cluster subsystem. Energy $E$ is given in $\mathrm{MeV}$ and the width of resonance states $\Gamma$ is in keV. Energy $E$ of a two-cluster system is determined with respect to the two-cluster threshold.

\begin{tabular}{|c|c|c|c|c|c|c|c|}
\hline \multirow[t]{2}{*}{$3 \mathrm{CC}$} & \multicolumn{4}{|c|}{ Binary subsystem } & \multicolumn{3}{|c|}{${ }^{8} \mathrm{Be}=\alpha+\alpha$} \\
\hline & $2 \mathrm{CC}$ & $J^{\pi}$ & E & $\Gamma$ & $J^{\pi}$ & E & $\Gamma$ \\
\hline${ }^{9} \mathrm{Be}$ & ${ }^{5} \mathrm{He}=\alpha+n$ & $3 / 2^{-}$ & 0.384 & 209 & $0^{+}$ & 0.859 & 958 \\
\hline${ }^{9} \mathrm{~B}$ & ${ }^{5} \mathrm{Li}=\alpha+p$ & $3 / 2^{-}$ & 1.236 & 725 & $0^{+}$ & 0.859 & 958 \\
\hline${ }^{10} \mathrm{~B}$ & ${ }^{6} \mathrm{Li}=\alpha+d$ & $1^{+}$ & -1.183 & & $0^{+}$ & 0.426 & 69 \\
\hline${ }^{11} \mathrm{~B}$ & ${ }^{7} \mathrm{Li}=\alpha+t$ & $3 / 2^{-}$ & -1.749 & & $0^{+}$ & 0.317 & 20 \\
\hline${ }^{11} \mathrm{C}$ & ${ }^{7} \mathrm{Be}=\alpha+{ }^{3} \mathrm{He}$ & $3 / 2^{-}$ & -0.941 & & $0^{+}$ & 0.317 & 20 \\
\hline
\end{tabular}

input parameters also underbind the energies of the ground states in ${ }^{6} \mathrm{Li},{ }^{7} \mathrm{Li}$, and ${ }^{7} \mathrm{Be}$. The largest difference between experimental and theoretical results is observed for ${ }^{7} \mathrm{Li}$ and equals $0.72 \mathrm{MeV}$, and the smallest difference equals $0.29 \mathrm{MeV}$ for ${ }^{6} \mathrm{Li}$.

Having determined the oscillator length $b$ and the parameter of the nucleon-nucleon forces, we have to select a part of the total Hilbert space which takes part in construction of the wave function of three-cluster continuous states. This part is restricted by the number of three-cluster channels $c$ and the number of hyperradial excitations or, in other words, the maximal number of oscillator shells. In all our calculations we use a standard set of the hyperspherical harmonics and hyperradial excitations. Positive parity states are calculated with the hyperspherical harmonics $K_{\min } \leqslant K \leqslant K_{\max }$, where $K_{\max }=14$ for the positive parity states and $K_{\max }=13$ for the negative parity states. The minimal value of the hypermomentum $K_{\min }$ equals the total orbital momentum $L$ for normal parity states $\pi=(-1)^{L}$ and $K_{\min }=L+1$ for the non-normal parity states. The total number of channels $N_{c h}$ depends on the total angular momentum $J$, the possible values of the total orbital moment $L$, and symmetry properties of the three-cluster system. To achieve the asymptotic region and to provide sufficient precision of our calculations, we take into account the hyperradial excitation up to 70 . This value of hyperradial excitation and the number of the hyperspherical channels cover a large range of intercluster distances and different shapes of the three-cluster triangle.

In this paper we will not discuss the dependence of parameters of resonance states on $K_{\max }$ and $N_{c h}$, and the convergence of the obtained results, as they were addressed in Refs. [15-18,22].

Within our models, the total spin $S$ of odd nuclei ${ }^{9} \mathrm{Be},{ }^{9} \mathrm{~B}$, ${ }^{11} \mathrm{~B}$, and ${ }^{11} \mathrm{C}$ equals $1 / 2$, thus the two following values of the total orbital momentum are involved in calculations:

$$
L=J-1 / 2, \quad J=L+1 / 2 .
$$

The total spin $S$ of the odd-odd nucleus ${ }^{10} \mathrm{~B}$ equals 1 , therefore bound and resonance states of the nucleus are constructed by three values of the total orbital momentum,

$$
L=J-1, \quad J=L, \quad J=L+1 .
$$

An interesting feature of description of ${ }^{10} \mathrm{~B}$ within the hyperspherical harmonics is that it includes almost two times more hyperspherical channels $c$ than nuclei ${ }^{9} \mathrm{Be},{ }^{9} \mathrm{~B},{ }^{11} \mathrm{~B}$, and ${ }^{11} \mathrm{C}$. Note that the coupling of states with different values of the total orbital momentum $L$ is totally determined by the spin-orbital interaction of nucleons.

\section{A. ${ }^{12} \mathrm{C}$ : Hoyle state}

In this section we are going to reexamine some results obtained in previous papers, concentrating our attention on properties of the Hoyle state in ${ }^{12} \mathrm{C}$.

In Table III we compare parameters of resonance states obtained within AMHHB [15] and CSM [36]. There are some consistencies in these two different methods of obtaining resonance states in the three-cluster continuum. Energy and total width of the first $0^{+}$resonance state (the Hoyle state) are 
TABLE III. Low-lying resonance states in ${ }^{12} \mathrm{C}$ calculated within the AMHHB and CSM.

\begin{tabular}{lccccc}
\hline \hline \multirow{2}{*}{$J^{\pi}$} & \multicolumn{2}{c}{ CSM [36] } & & \multicolumn{2}{c}{ AMHHB [15] } \\
\cline { 2 - 3 } \cline { 5 - 6 } & $E(\mathrm{MeV})$ & $\Gamma(\mathrm{keV})$ & & $E(\mathrm{MeV})$ & $\Gamma(\mathrm{keV})$ \\
\hline $0^{+}$ & 0.76 & 2.4 & & 0.68 & 2.9 \\
& 1.66 & 1480 & & 5.16 & 534 \\
$2^{+}$ & 2.28 & 1100 & & 2.78 & 10 \\
& 5.14 & 1900 & & 3.17 & 280 \\
& 6.82 & 240 & & 5.60 & 0.6 \\
$1^{-}$ & 3.65 & 0.30 & & 3.52 & 0.21 \\
$3^{-}$ & 1.51 & $2.0 \times 10^{-3}$ & & 0.67 & 8.34 \\
\hline \hline
\end{tabular}

very close in both methods. The same is observed for other narrow $1^{-}$resonance states in ${ }^{12} \mathrm{C}$.

In Fig. 1 we display the weights $W_{\text {sh }}$ which represent the structure of the wave function of the Hoyle state. As we see, the weights of oscillator shells have very large amplitudes, and the main contribution to the wave function in the internal region comes from the oscillator shells $0 \leqslant N_{\mathrm{sh}} \leqslant 30$. In the asymptotic region, this function has an oscillatory behavior with much smaller amplitude. We consider such a behavior of a resonance wave function as a "standard" or pattern for the Hoyle-analog states.

It is interesting to compare the wave function of the Hoyle state with wave functions of other resonance states in ${ }^{12} \mathrm{C}$. We selected the second $0^{+}$resonance state and $1^{-}$resonance state. As it follows from Table III, the second $0^{+}$resonance state is a broad resonance state $(\Gamma=534 \mathrm{keV})$ while the $1^{-}$ resonance state is a narrow resonance state $(\Gamma=0.21 \mathrm{keV})$. The presented weights $W_{\text {sh }}$ for these two states (Fig. 2) demonstrate that the wave function of the narrow $1^{-}$state has a behavior which is close to the standard behavior of the Hoyle state, as it has very large amplitudes of the oscillator shells $0 \leqslant N_{\text {sh }} \leqslant 30$. Contrary to this case, the wave function of the second resonance $0^{+}$state has rather small amplitudes of the

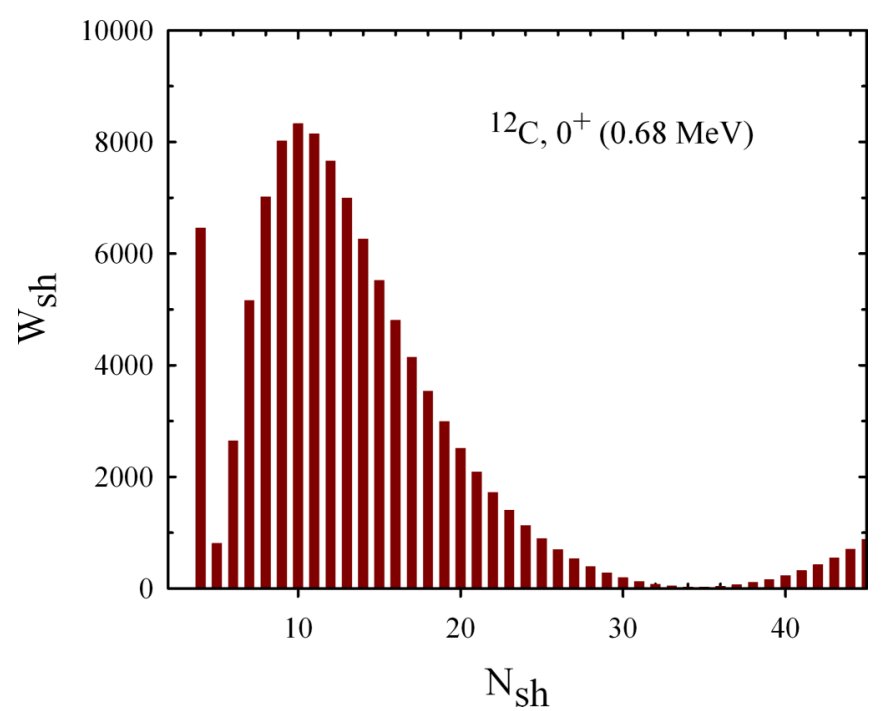

FIG. 1. Weights of different oscillator shells in the wave function of the first $0^{+}$resonance state in ${ }^{12} \mathrm{C}$.

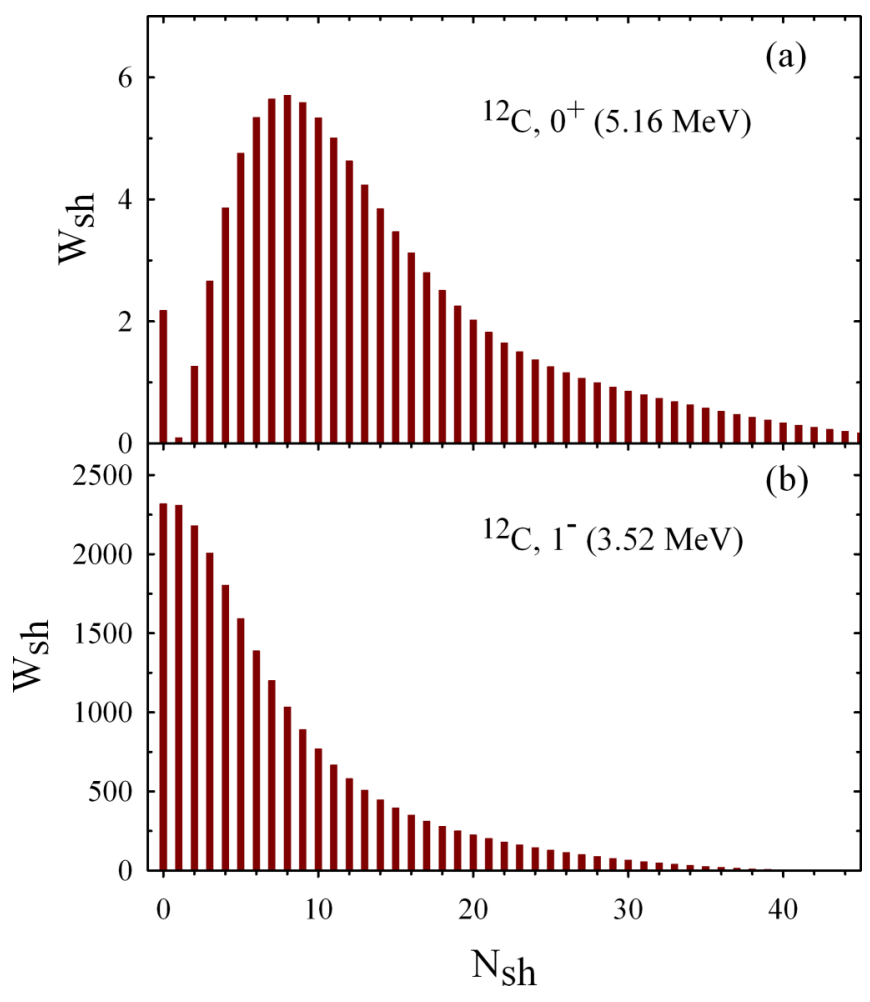

FIG. 2. Structure of wave functions of the second $0^{+}$(a) and first $1^{-}$(b) resonance states in ${ }^{12} \mathrm{C}$.

lowest oscillator shells. It is naturally to assume that the $1^{-}$ resonance state is the Hoyle-analog state in ${ }^{12} \mathrm{C}$. We will use the standard behavior of the wave function of the Hoyle state, displayed in Fig. 1, as the additional criterion for selecting the Hoyle-analog states.

In Fig. 3 we compare the resonance wave function with the wave function of the pseudo-bound state, which was calculated in the bound state approximation with $N_{\max }=70$.

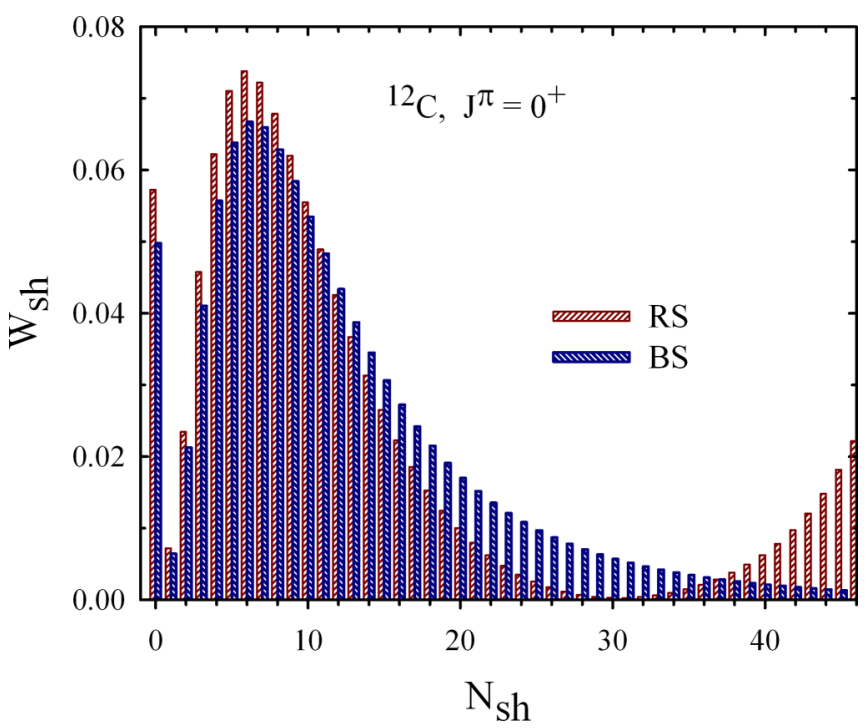

FIG. 3. Comparing wave functions of the $0^{+}$resonance state (RS) and the $0^{+}$pseudo-bound state (BS) in ${ }^{12} \mathrm{C}$. 
TABLE IV. The energy, width and average distances $R_{1}, R_{2}$ between clusters for the ground states and for the $0^{+}$and $1^{-}$resonance states in ${ }^{12} \mathrm{C}$.

\begin{tabular}{lrccc}
\hline \hline$J^{\pi}$ & $E(\mathrm{MeV})$ & $\Gamma(\mathrm{keV})$ & $R_{1}(\mathrm{fm})$ & $R_{2}(\mathrm{fm})$ \\
\hline $0^{+}$ & -11.37 & & 3.12 & 3.60 \\
& 0.68 & 2.9 & 6.95 & 8.02 \\
& 5.16 & 534 & 6.43 & 7.43 \\
$1^{-}$ & 3.52 & 0.21 & 6.07 & 7.00 \\
\hline \hline
\end{tabular}

Both states have approximately the same energy; the energy of the resonance state is $0.536 \mathrm{MeV}$, while the pseudo-bound state has the energy $0.529 \mathrm{MeV}$.

It is worthwhile to notice that approximately such a structure of the Hoyle state wave function has been obtained within the complex scaling method in Ref. [37] and within fermion molecular dynamics in Ref. [38].

We determined the shape of the triangle composed of three $\alpha$ particles in bound and resonance states. The average distances between clusters are displayed in Table IV.

The shape and size of triangles for the ground and the first $0^{+}$ resonance states are consistent with the corresponding density distributions displayed in Figs. 8 and 9 of Ref. [15]. It is interesting to note that the shape of resonance states, shown in Table IV, is almost independent of the energy and total width of the resonance state, and the structure of resonance wave functions shown in Figs. 1 and 2. The main conclusion one may deduce from Table IV is that the average distances between $\alpha$ particles are rather large. The ground state of ${ }^{12} \mathrm{C}$ shows a compact three-cluster configuration, as expected.

Having reanalyzed properties of the Hoyle state and other resonance states in ${ }^{12} \mathrm{C}$, we suggest the following criteria for the Hoyle-analog states:

(1) The Hoyle-analog state is a very narrow resonance state in the three-cluster continuum.

(2) The wave function of the Hoyle-analog state has large values of amplitudes $W_{\text {sh }}$ in the internal region.

As we pointed out above, we consider the first criterion is the most important one. We believe that the more long-lived resonance state has more chances for the system to transit from a resonance state into a bound states, and vice versa. It is well-known that a resonance state could substantially increase a cross section of a process if the total width of this resonance state is very small. To quantify the "narrowness" of a resonance state we will calculate the ratio $\Gamma / E$. For the original Hoyle state this ratio is $2.24 \times 10^{-7}$. Such resonance states are also called quasistationary states. As an additional and important criterion we will use the behavior of the weights $W_{\text {sh }}$ of oscillator shells in the wave function of the resonance state.

Considering candidates of the Hoyle-analog states, we are also going to check other criteria formulated in the Introduction.

\section{B. ${ }^{9}$ Be and ${ }^{9} \mathrm{~B}$}

As was pointed out above, spectra of resonance states in ${ }^{9} \mathrm{Be}$ and ${ }^{9} \mathrm{~B}$ have been investigated within the present model
TABLE V. Spectra of resonance states of ${ }^{9} \mathrm{Be}$ and ${ }^{9} \mathrm{~B}$ calculated within the AMHHB model with MHNP.

\begin{tabular}{lrrrrrc}
\hline \hline \multicolumn{3}{c}{${ }^{9} \mathrm{Be}$} & & & \multicolumn{3}{c}{${ }^{9} \mathrm{~B}$} \\
\cline { 1 - 2 } \cline { 5 - 6 }$J^{\pi}$ & $E(\mathrm{MeV})$ & $\Gamma(\mathrm{MeV})$ & & $J^{\pi}$ & $E(\mathrm{MeV})$ & $\Gamma(\mathrm{MeV})$ \\
\hline $3 / 2^{-}$ & -1.574 & & & $3 / 2^{-}$ & 0.379 & $1.08 \times 10^{-6}$ \\
$1 / 2^{+}$ & 0.338 & 0.17 & & $1 / 2^{+}$ & 0.636 & 0.48 \\
$5 / 2^{-}$ & 0.897 & $2.36 \times 10^{-5}$ & & $5 / 2^{-}$ & 2.805 & 0.02 \\
$5 / 2^{+}$ & 2.086 & 0.11 & & $3 / 2^{+}$ & 2.338 & 2.80 \\
$3 / 2^{-}$ & 2.704 & 2.53 & & $1 / 2^{-}$ & 3.398 & 3.43 \\
$1 / 2^{-}$ & 2.866 & 1.60 & & $5 / 2^{+}$ & 3.670 & 0.42 \\
$3 / 2^{+}$ & 4.062 & 1.22 & & $3 / 2^{-}$ & 3.420 & 3.36 \\
$7 / 2^{-}$ & 4.766 & 4.04 & & $5 / 2^{-}$ & 5.697 & 5.15 \\
$9 / 2^{+}$ & 4.913 & 1.27 & & $9 / 2^{+}$ & 6.503 & 2.01 \\
$5 / 2_{2}^{-}$ & 5.365 & 4.38 & & $7 / 2^{-}$ & 6.779 & 0.90 \\
\hline \hline
\end{tabular}

in Refs. [22] and [16]. In Ref. [16] we have discovered several resonance states that can be considered as the Hoyle-analog states. For completeness of the explanation we briefly present the main results relevant to the subject of the present paper.

Energies and widths of the resonance states in ${ }^{9} \mathrm{Be}$ and ${ }^{9} \mathrm{~B}$ presented in Ref. [16] were obtained with the modified version of the Hasegawa-Nagata potential, which is often used in calculations of two- and three-cluster structures of light nuclei. It was shown that our three-cluster model with such a potential reproduces fairly good spectra of resonance states in both nuclei. It was also demonstrated that the Hasegawa-Nagata potential provides a more adequate description of resonance states in ${ }^{9} \mathrm{Be}$ and ${ }^{9} \mathrm{~B}$ than the Minnesota potential (see details in Ref. [22]).

In Table $\mathrm{V}$ we collect energies and widths of resonance states in ${ }^{9} \mathrm{Be}$ and ${ }^{9} \mathrm{~B}$.

There is only one very narrow resonance state in each nucleus. This is the $5 / 2^{-}$resonance state in ${ }^{9} \mathrm{Be}$ and the $3 / 2^{-}$resonance state in ${ }^{9} \mathrm{~B}$, which is the "ground state" of the nucleus. We considered these resonance states to be candidates for the Hoyle-analog states. We also added the $1 / 2^{+}$resonance states to that list of resonance states, as they lie close to the three-cluster threshold. Other resonance states in ${ }^{9} \mathrm{Be}$ and ${ }^{9} \mathrm{~B}$ have a large total width and they were disregarded.

In Fig. 4 we display the structure of wave functions of the $5 / 2^{-}$resonance states in ${ }^{9} \mathrm{Be}$ and ${ }^{9} \mathrm{~B}$. The total width of the $5 / 2^{-}$resonance states in ${ }^{9} \mathrm{Be}$ is $24 \mathrm{eV}$ and amplitudes of the dominant shell weights $W_{\mathrm{sh}}$ are of $10^{5}$ order of magnitude. The same resonance state in ${ }^{9} \mathrm{~B}$ is wider $(\Gamma=18 \mathrm{keV})$ and thus amplitudes of the dominant shell weights $W_{\mathrm{sh}}$ are less than 1000 . One can see that the oscillator shells with $0 \leqslant N_{\text {sh }}<20$ give the main contribution to the wave functions of the $5 / 2^{-}$ resonance states. In Fig. 4 we also display $W_{\text {sh }}$ in a logarithmic scale to demonstrate their behavior in the internal region. Within the internal region, wave functions are decreasing exponentially like wave functions of bound states. Such a behavior of wave functions of the $5 / 2^{-}$resonance states in ${ }^{9} \mathrm{Be}$ and ${ }^{9} \mathrm{~B}$ allows us to consider these resonance states as the Hoyle-analog states.

In Ref. [16] we have also considered the $1 / 2^{+}$resonance states in ${ }^{9} \mathrm{Be}$ and ${ }^{9} \mathrm{~B}$ as possible candidates to the Hoyle-analog states. These resonances lie very close to the three-cluster 


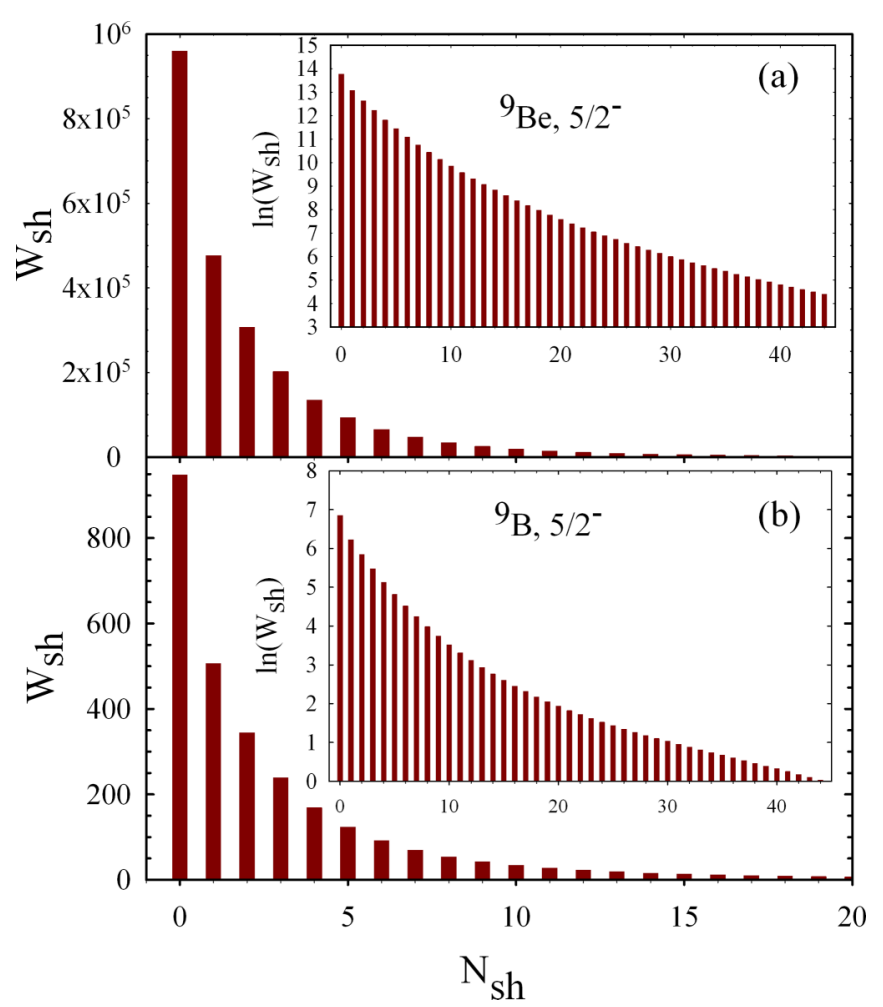

FIG. 4. Weight $W_{\mathrm{sh}}$ as a function of oscillator shell $N_{\mathrm{sh}}$ for the $5 / 2^{-}$resonance state in ${ }^{9} \mathrm{Be}(\mathrm{a})$ and ${ }^{9} \mathrm{~B}(\mathrm{~b})$.

threshold; however, the $1 / 2^{+}$resonance states are rather wide resonances and their wave functions both in coordinate and oscillator representations indicate a very dispersed three-cluster configuration (see the wave functions in coordinate space in Fig. 5). The latter result is also confirmed by the average distances $R_{1}$ and $R_{2}$.

We also analyze all resonance states in ${ }^{9} \mathrm{Be}$ in order to find the Hoyle-analog state. We suggested that the $5 / 2^{-}$resonance

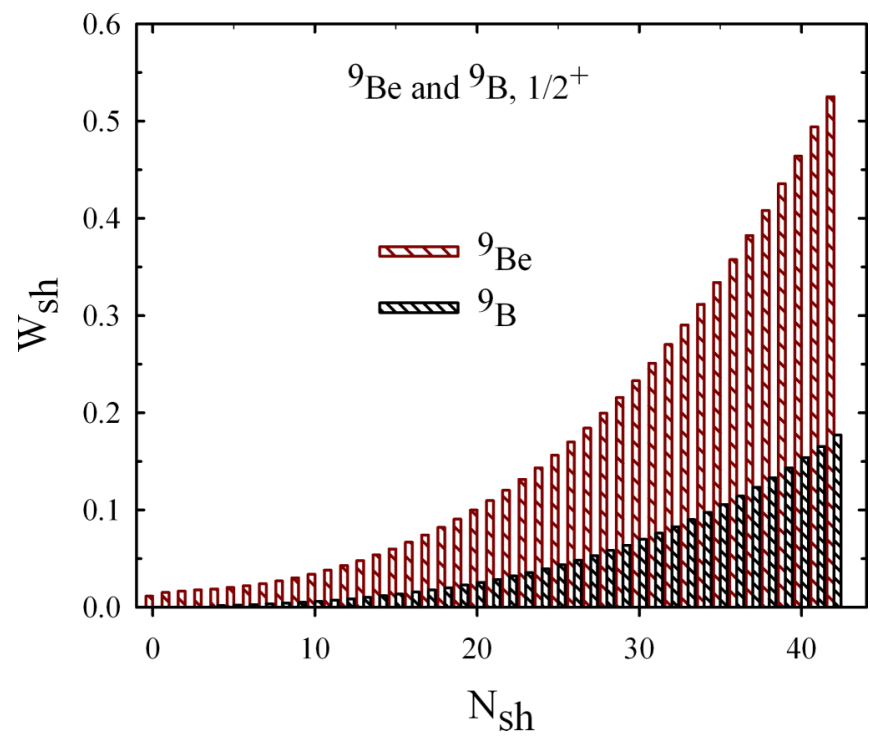

FIG. 5. Weights of different oscillator shells in wave functions of the $1 / 2^{+}$resonance states in ${ }^{9} \mathrm{Be}$ (red color) and ${ }^{9} \mathrm{~B}$ (blue color).
TABLE VI. The spectrum of positive- and negative-parity resonance states in ${ }^{11} \mathrm{~B}$. Energy is in $\mathrm{MeV}$ and measured from the three-cluster $\alpha+\alpha+t$ threshold.

\begin{tabular}{lccccc}
\hline \hline$J^{\pi}$ & $E(\mathrm{MeV})$ & $\Gamma(\mathrm{keV})$ & $J^{\pi}$ & $E(\mathrm{MeV})$ & $\Gamma(\mathrm{keV})$ \\
\hline $3 / 2^{-}$ & 0.755 & 0.58 & $1 / 2^{+}$ & 0.437 & 15.26 \\
& 1.402 & 185.18 & & 0.702 & 12.30 \\
& 1.756 & 143.72 & & 1.597 & 15.95 \\
$1 / 2^{-}$ & 1.436 & 374.64 & $3 / 2^{+}$ & 1.147 & 1.498 \\
& 1.895 & 100.95 & & 1.367 & 8.58 \\
& 2.404 & 450.07 & & 1.715 & 41.24 \\
$5 / 2^{-}$ & 0.583 & $5.14 \times 10^{-4}$ & $5 / 2^{+}$ & 1.047 & 1.54 \\
& 1.990 & 32.63 & & 1.951 & 40.20 \\
& 2.251 & 138.87 & & 2.265 & 54.73 \\
& 2.905 & 120.46 & & 2.748 & 167.61 \\
$7 / 2^{-}$ & 1.591 & 4.14 & $7 / 2^{+}$ & 1.076 & $2.04 \times 10^{-2}$ \\
& 1.778 & 3.04 & & 2.119 & 26.32 \\
& 2.471 & 20.18 & & 2.536 & 100.47 \\
\hline \hline
\end{tabular}

state can be considered as the Hoyle-analog state as this is a very narrow resonance state. It lives long enough and may transform to the $3 / 2^{-}$ground state of ${ }^{9} \mathrm{Be}$ by emitting the quadrupole $\gamma$ quanta. This reaction, which involves the triple collision of two $\alpha$ particles and a neutron and a subsequent radiation of $\gamma$ quanta, can be considered as an additional path for the synthesis of ${ }^{9} \mathrm{Be}$ nuclei.

It was shown in Ref. [16] that such behavior of the wave function (Fig. 5) is typical for a low-lying resonance state with a relatively large value of the total width.

\section{C. ${ }^{11} \mathrm{~B}$ and ${ }^{11} \mathrm{C}$}

Now we consider the spectra of resonance states in ${ }^{11} \mathrm{~B}$ and ${ }^{11} \mathrm{C}$. In Table VI we display the energy and width of resonance states in the three-cluster $\alpha+\alpha+t$ continuum of ${ }^{11} \mathrm{~B}$, which were calculated in Ref. [17].

In a small range of energies $0<E<3 \mathrm{MeV}$ we observed 26 resonance states. The large part of these resonances are narrow resonance states with the total width less than $50 \mathrm{keV}$. A similar picture is observed in ${ }^{11} \mathrm{C}$. The energy and width of positive- and negative-parity states are shown in Table VII. Details of these calculations can be found in Ref. [17].

By using the criteria for selecting the candidates for the Hoyle-analog states, formulated above, we selected four resonance states in ${ }^{11} \mathrm{~B}$ and four resonance states in ${ }^{11} \mathrm{C}$. In Table VIII we display the properties of the selected resonance states in ${ }^{11} \mathrm{~B}$ and ${ }^{11} \mathrm{C}$, and compare them with some bound states. We did not include the $1 / 2^{+}$resonance state in ${ }^{11} \mathrm{C}$ as it has a relatively large total width.

Figures 6 and 7 demonstrating wave functions of the 5/2resonance states in ${ }^{11} \mathrm{~B}$ and ${ }^{11} \mathrm{C}$ explicitly indicate that these resonance states can be considered as the Hoyle-analog state. Both resonance states have very large amplitudes of weights $W_{\mathrm{sh}}$. The structure of the wave functions of the $5 / 2^{-}$resonance states in ${ }^{11} \mathrm{C}$ looks like a wave function of a bound state. These results also show that the average distances between clusters $R_{1}$ and $R_{2}$ in these resonance states are very close to average distances for bound states, for instance, for the first excited 
TABLE VII. The spectrum of positive- and negative-parity resonance states in ${ }^{11} \mathrm{C}$. Energy is in $\mathrm{MeV}$ and measured from the three-cluster $\alpha+\alpha+{ }^{3} \mathrm{He}$ threshold.

\begin{tabular}{lccccc}
\hline \hline$J^{\pi}$ & $E(\mathrm{MeV})$ & $\Gamma(\mathrm{keV})$ & $J^{\pi}$ & $E(\mathrm{MeV})$ & $\Gamma(\mathrm{keV})$ \\
\hline $3 / 2^{-}$ & 0.805 & $9.93 \times 10^{-3}$ & $1 / 2^{+}$ & 0.906 & 162.94 \\
& 1.920 & 105.08 & & 1.930 & 59.88 \\
& 2.324 & 619.76 & & 2.679 & 86.69 \\
$1 / 2^{-}$ & 1.142 & 0.708 & $3 / 2^{+}$ & 2.268 & 34.25 \\
& 2.266 & 790.98 & & 2.478 & 159.28 \\
& 3.014 & 366.15 & & 2.850 & 115.19 \\
$5 / 2^{-}$ & 0.783 & $9.64 \times 10^{-5}$ & $5 / 2^{+}$ & 1.460 & 0.90 \\
& 1.897 & 5.77 & & 2.346 & 82.72 \\
& 3.026 & 182.69 & & 3.179 & 122.75 \\
& 3.491 & 392.96 & $7 / 2^{+}$ & 1.765 & $7.40 \times 10^{-2}$ \\
$7 / 2^{-}$ & 2.700 & 66.63 & & 2.542 & 8.19 \\
& 3.538 & 21.18 & & 3.237 & 119.13 \\
\hline \hline
\end{tabular}

$3 / 2^{-}$state in ${ }^{11} \mathrm{C}$. From the average distances $R_{1}$ and $R_{2}$ for the resonance states in ${ }^{11} \mathrm{~B}$ and ${ }^{11} \mathrm{C}$ in Table VIII, we see that the most narrow $5 / 2^{-}$resonance state in ${ }^{11} \mathrm{C}$ has the most compact configuration of three clusters. In contrast to this resonance state, the narrowest $5 / 2^{-}$resonance state at $E=0.583 \mathrm{MeV}$ in ${ }^{11} \mathrm{~B}$, the total width of which is five time larger than the width of the $5 / 2^{-}$resonance state in ${ }^{11} \mathrm{C}$, has a rather dispersed structure with the average distance between $\alpha$ particles being equal to $7.2 \mathrm{fm}$.

It is interesting to note that the Coulomb interaction makes the $5 / 2^{-}$resonance state in ${ }^{11} \mathrm{C}$ more narrow than that in ${ }^{11} \mathrm{~B}$. As one may expect, it also increases the energy of the resonance in ${ }^{11} \mathrm{C}$ comparing to its position in ${ }^{11} \mathrm{~B}$. The same picture is observed for the $3 / 2^{-}$resonance states in ${ }^{11} \mathrm{~B}$ and ${ }^{11} \mathrm{C}$. The larger Coulomb barrier in ${ }^{11} \mathrm{C}$ leads to the very large amplitudes of $W_{\mathrm{sh}}$ for the $3 / 2^{-}$resonances state. One can compare amplitudes of $W_{\mathrm{sh}}$ for $3 / 2^{-}$resonance states in ${ }^{11} \mathrm{~B}$ and ${ }^{11} \mathrm{C}$ in Figs. 8 and 9, respectively.

We do not show wave functions of the $1 / 2^{+}$resonance states in ${ }^{11} \mathrm{~B}$ and ${ }^{11} \mathrm{C}$ here as they are very similar to the wave

TABLE VIII. Parameters of resonance states in ${ }^{11} \mathrm{~B}$ and ${ }^{11} \mathrm{C}$ selected as candidates to the Hoyle-analog states. The average distances $R_{1}$ and $R_{2}$ are presented both for resonance states and for bound states.

\begin{tabular}{|c|c|c|c|c|c|c|}
\hline Nucleus & $J^{\pi}$ & $E(\mathrm{MeV})$ & $\Gamma(\mathrm{keV})$ & $\Gamma / E$ & $R_{1}(\mathrm{fm})$ & $R_{2}(\mathrm{fm})$ \\
\hline \multirow[t]{7}{*}{${ }^{11} \mathrm{~B}$} & $3 / 2^{-}$ & -11.055 & & & 2.60 & 2.88 \\
\hline & $3 / 2^{-}$ & -5.667 & & & 2.90 & 3.38 \\
\hline & $3 / 2^{-}$ & -0.589 & & & 4.83 & 6.79 \\
\hline & $1 / 2^{+}$ & 0.437 & 15.26 & $3.49 \times 10^{-2}$ & 10.48 & 6.77 \\
\hline & $5 / 2^{-}$ & 0.583 & $5.14 \times 10^{-4}$ & $8.81 \times 10^{-7}$ & 4.71 & 7.20 \\
\hline & $3 / 2^{-}$ & 0.755 & 0.58 & $7.7 \times 10^{-4}$ & 5.36 & 7.75 \\
\hline & $5 / 2^{+}$ & 1.047 & 1.54 & $1.47 \times 10^{-3}$ & 4.98 & 7.47 \\
\hline \multirow[t]{6}{*}{${ }^{11} \mathrm{C}$} & $3 / 2^{-}$ & -9.073 & & & 2.64 & 2.90 \\
\hline & $3 / 2^{-}$ & -3.835 & & & 2.97 & 3.43 \\
\hline & $1 / 2^{+}$ & 0.906 & 162.94 & & 10.75 & 7.08 \\
\hline & $5 / 2^{-}$ & 0.783 & $9.64 \times 10^{-5}$ & $1.23 \times 10^{-7}$ & 3.20 & 3.87 \\
\hline & $3 / 2^{-}$ & 0.805 & $9.93 \times 10^{-3}$ & $1.23\left\{\times 10^{-5}\right.$ & 5.02 & 6.86 \\
\hline & $5 / 2^{+}$ & 1.460 & 0.90 & $6.16 \times 10^{-4}$ & 5.00 & 6.69 \\
\hline
\end{tabular}

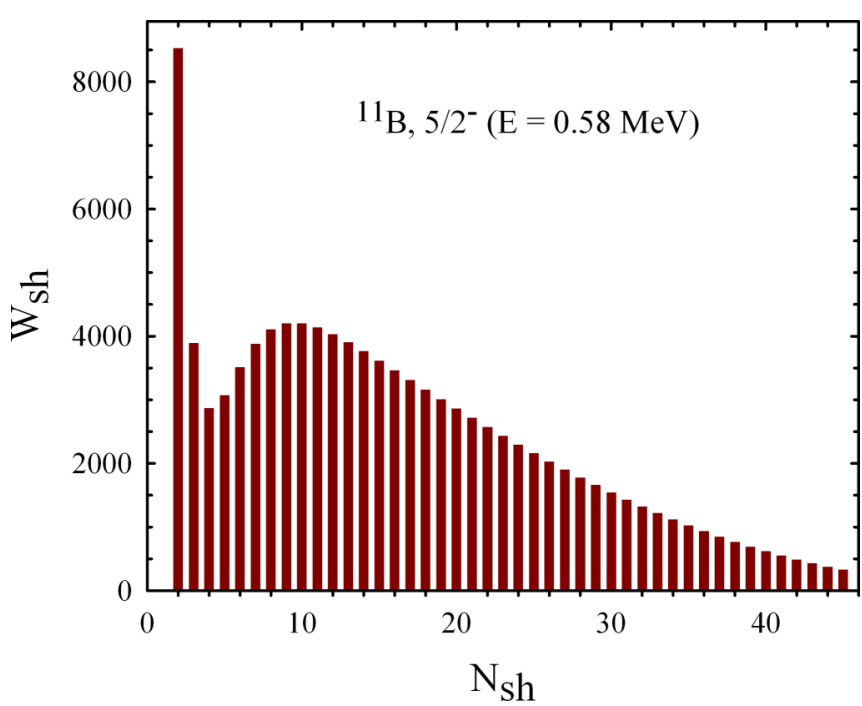

FIG. 6. Weights of oscillator shells in the wave functions of the $5 / 2^{-}$resonance state $(E=0.58 \mathrm{MeV} \Gamma=0.5 \mathrm{eV})$ in ${ }^{11} \mathrm{~B}$.

functions of these resonance states in ${ }^{9} \mathrm{Be}$ and ${ }^{9} \mathrm{~B}$. Moreover, the shape of three-cluster triangles in those pairs of nuclei is also similar.

As we pointed out in the Introduction, there are few publications which are devoted to the Hoyle-analog states in ${ }^{11} \mathrm{~B}$ and ${ }^{11} \mathrm{C}$. In Refs. [6] and [8], the spectra of ${ }^{11} \mathrm{~B}$ and ${ }^{11} \mathrm{C}$ were obtained within the antisymmetrized molecular dynamics (AMD) model. The excited states have been treated as bound states, which means that the widths and energies of these states with respect to the three-cluster thresholds were not determined. By analyzing the probability of electromagnetic transitions, the authors came to the conclusion that the $3 / 2^{-}$ excited states have dilute cluster structures $\alpha+\alpha+t$ and $\alpha+\alpha+{ }^{3} \mathrm{He}$, and thus can be considered as the Hoyle-analog states. It was also claimed by the authors that the $5 / 2^{-}$states do not have a well-developed cluster structure and therefore cannot be considered as the Hoyle-analog states.

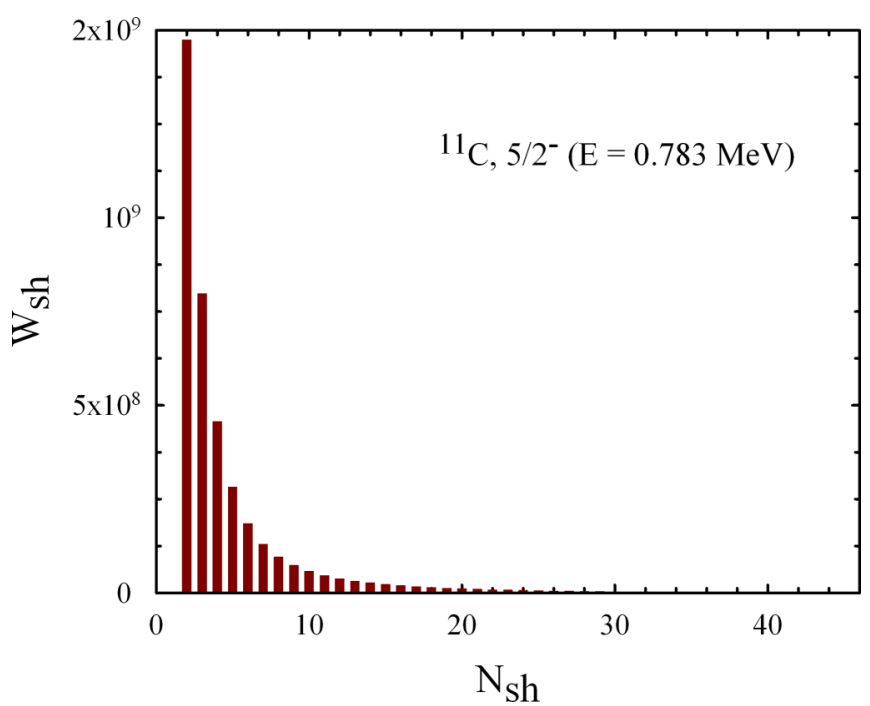

FIG. 7. Structure of the wave function of the very narrow $5 / 2^{-}$ resonance state in ${ }^{11} \mathrm{C}$. 


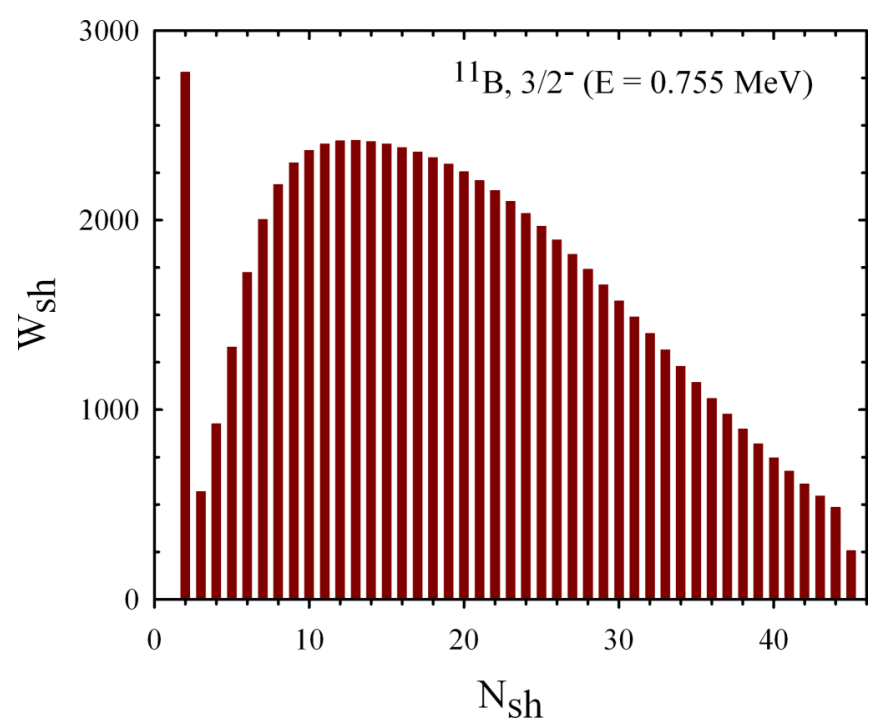

FIG. 8. The shape of the wave function of the $3 / 2^{-}$resonance state in ${ }^{11} \mathrm{~B}$.

In Refs. [7] and [10], resonance states in the two- and three-body continuum of ${ }^{11} \mathrm{~B}$ and ${ }^{11} \mathrm{C}$ were determined with the complex scaling method. The $3 / 2^{-}$resonance state is located below the $\alpha+\alpha+t$ threshold and has a compact cluster configuration, as was shown by the authors of Refs. [7,10], and therefore was not considered as a candidate for the Hoyleanalog states. The wave function of the $1 / 2^{+}$resonance state, which has "the gas-like structure with a large nuclear radius," as stressed by the authors, thus can be considered as the Hoyle-analog state. It is interesting to note that the parameters ( $E=0.75 \mathrm{MeV}, \Gamma=190 \mathrm{keV}$ ) of the $1 / 2^{+}$resonance state, determined in Refs. [7,10], are rather different from those ( $E=0.44 \mathrm{MeV}, \Gamma=15 \mathrm{keV}$ ) displayed in the present paper. This difference can be ascribed to the different types of nucleon-nucleon potentials involved in these two calculations. The $1 / 2^{+}$resonance state, obtained in our calculations, has

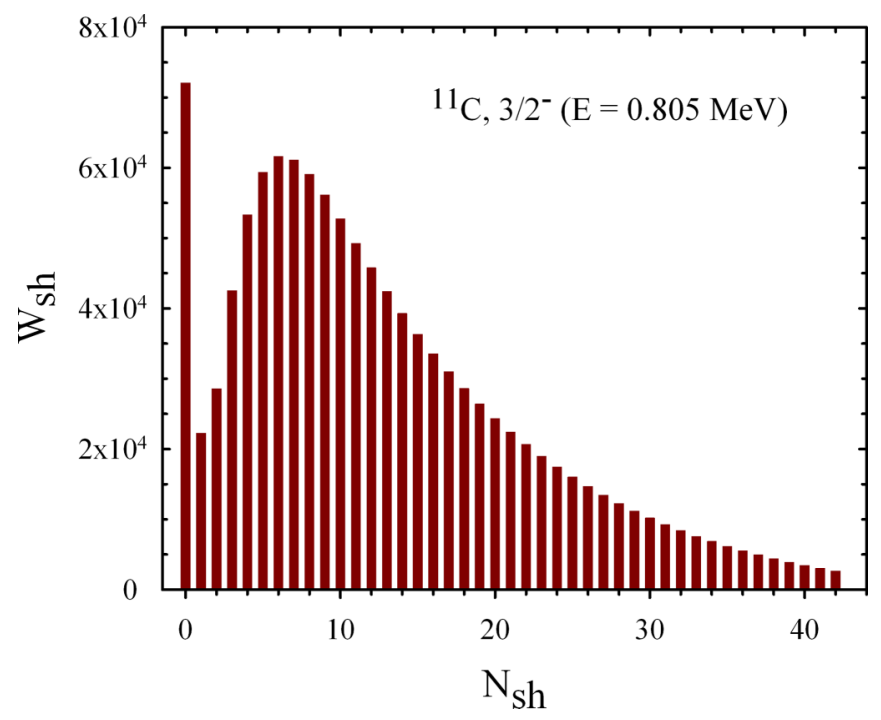
in ${ }^{11} \mathrm{C}$.
TABLE IX. Parameters of resonance states in ${ }^{10} \mathrm{~B}$. The average distances $R_{1}$ and $R_{2}$ are calculated for the candidates for the Hoyleanalog states.

\begin{tabular}{lllccc}
\hline \hline$J^{\pi}$ & $E(\mathrm{MeV})$ & $\Gamma(\mathrm{keV})$ & $\Gamma / E$ & $R_{1}(\mathrm{fm})$ & $R_{2}(\mathrm{fm})$ \\
\hline $1^{+}$ & 0.604 & 232.30 & 0.384 & & \\
& 0.987 & 7.08 & $7.17 \times 10^{-3}$ & 6.67 & 10.67 \\
& 1.536 & 196.36 & 0.128 & & \\
$2^{+}$ & 1.055 & 12.063 & $11.43 \times 10^{-3}$ & 6.64 & 10.83 \\
& 2.810 & 170.74 & $60.76 \times 10^{-3}$ & & \\
$3^{+}$ & 1.062 & 11.73 & $11.05 \times 10^{-3}$ & 6.43 & 10.35 \\
& 2.202 & 526.47 & 0.239 & & \\
$1^{-}$ & 1.100 & 76.75 & $69.77 \times 10^{-3}$ & 9.31 & 10.84 \\
& 1.820 & 562.71 & 0.309 & & \\
\hline \hline
\end{tabular}

also a large nuclear radius; however, it is not considered as the Hoyle-analog state under our criterion.

\section{D. ${ }^{10} \mathrm{~B}$}

In Table IX we show the three-cluster resonance states in ${ }^{10} \mathrm{~B}$ calculated with the MP. Details of these calculations can be found in Ref. [18], where the spectrum of bound states of ${ }^{10} \mathrm{~B}$ has been discussed. Here, we use the same input parameters to calculate the spectrum of resonance states in the three-cluster $\alpha+\alpha+d$ continuum. As we can see in Table IX, there are a few narrow resonance states which can be considered as the Hoyle-analog states. Three resonance states have a total width less than $12 \mathrm{keV}$, and the ratio $\Gamma / E$ does not exceed $11.5 \times 10^{-3}$.

In Table IX we also show the average distances between interacting clusters. It is necessary to recall that $R_{2}$ stands for the distance between two $\alpha$ particles, and $R_{1}$ denotes the distance between the deuteron and the center of mass of two $\alpha$ particles. It is interesting to compare the average distance between clusters for resonance states with those for the bound states. For the ground $3^{+}$state we obtained $R_{1}=2.60 \mathrm{fm}$ and $R_{2}=3.10 \mathrm{fm}$. This is a compact configuration despite the fact that the binding energy is $-5.95 \mathrm{MeV}$ accounted from the three-cluster threshold $\alpha+\alpha+d$, which is not very small. The first excited $3^{+}$state is a weakly bound state as its energy is $-0.95 \mathrm{MeV}$; however, it is also a rather compact configuration with the average distances $R_{1}=4.07$ and $R_{1}=$ $5.35 \mathrm{fm}$. As we see in Table IX, all resonance states selected as the candidates for the Hoyle-analog states have a dispersed configuration with a large distance between $\alpha$ particles.

Let us turn our attention to the wave functions of the selected resonance states. In Fig. 10 we display shell weights in wave functions of the narrow $3^{+}$and $1^{+}$resonance states in ${ }^{10} \mathrm{~B}$. These resonance state have the smallest total width among all resonances in ${ }^{10} \mathrm{~B}$. One notices that the compact three-cluster configuration $\left(N_{\mathrm{sh}}=0\right)$ has a relatively large contribution to these wave functions. The shapes of the curves are similar to the shape of the Hoyle state (Fig. 1); however, the amplitudes are much more smaller.

We assume that the interplay of the attractive potential, created by the central and spin-orbital parts of the nucleonnucleon interaction, and the repulsive potential, formed by the Coulomb interaction, does not create a favorable situation for very narrow resonance states in ${ }^{10} \mathrm{Be}$. 


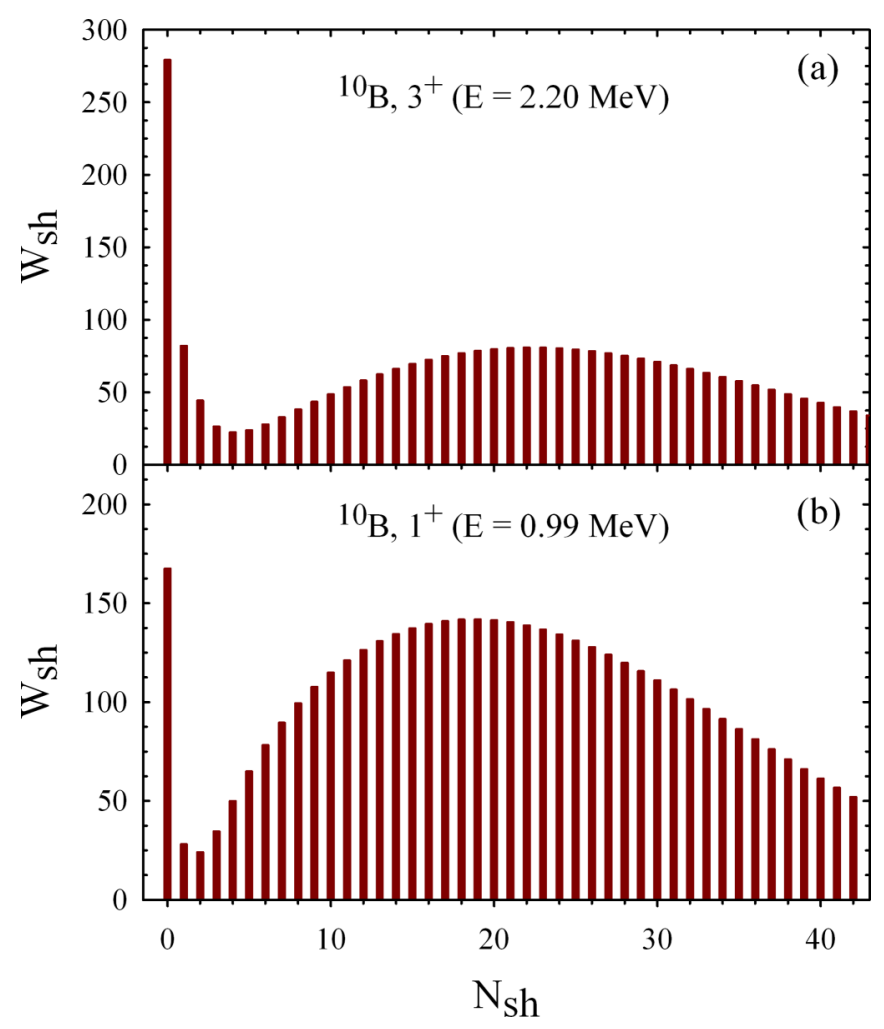

FIG. 10. Weights of different oscillator shells in wave functions of the $3^{+}$(a) and $1^{+}$(b) resonance states in ${ }^{10} \mathrm{~B}$.

\section{CONCLUSION}

We have performed a systematic investigation of the threecluster resonance states in light nuclei ${ }^{9} \mathrm{Be},{ }^{9} \mathrm{~B},{ }^{10} \mathrm{~B},{ }^{11} \mathrm{~B}$, ${ }^{11} \mathrm{C}$, and ${ }^{12} \mathrm{C}$. These nuclei have been considered to have a three-cluster structure composed of two $\alpha$ particles and an $s$-shell nucleus. A microscopic three-cluster model was applied to search for and to study resonance states embedded in the three-cluster continuum. This model imposes proper boundary conditions by employing hyperspherical coordinates and hyperspherical harmonics. Having reanalyzed properties of the Hoyle state, we formulated criteria for the Hoyle-analog states. Among these resonances, we found the Hoyle-analog states in these nuclei. The Hoyle-analog states are created by

TABLE X. Parameters of the Hoyle-analog states in light nuclei ${ }^{9} \mathrm{Be},{ }^{9} \mathrm{~B},{ }^{11} \mathrm{~B}$, and ${ }^{11} \mathrm{C}$.

\begin{tabular}{lccccc}
\hline \hline Nucleus & Configuration & $J^{\pi}$ & $E(\mathrm{MeV})$ & $\Gamma(\mathrm{keV})$ & $\Gamma / E$ \\
\hline${ }^{9} \mathrm{Be}$ & $\alpha+\alpha+n$ & $5 / 2^{-}$ & 0.897 & $2.36 \times 10^{-2}$ & $2.63 \times 10^{-5}$ \\
${ }^{9} \mathrm{~B}$ & $\alpha+\alpha+n$ & $3 / 2^{-}$ & 0.379 & $1.08 \times 10^{-3}$ & $2.84 \times 10^{-6}$ \\
& & $5 / 2^{-}$ & 2.805 & $18.0 \times 10^{-3}$ & $6.42 \times 10^{-6}$ \\
${ }^{11} \mathrm{~B}$ & $\alpha+\alpha+{ }^{3} \mathrm{H}$ & $5 / 2^{-}$ & 0.583 & $5.14 \times 10^{-4}$ & $8.87 \times 10^{-7}$ \\
& & $3 / 2^{-}$ & 0.755 & 0.58 & $7.70 \times 10^{-4}$ \\
& & $5 / 2^{+}$ & 1.047 & 1.54 & $1.47 \times 10^{-3}$ \\
${ }^{11} \mathrm{C}$ & $\alpha+\alpha+{ }^{3} \mathrm{He}$ & $5 / 2^{-}$ & 0.783 & $9.64 \times 10^{-5}$ & $1.23 \times 10^{-7}$ \\
& & $3 / 2^{-}$ & 0.805 & $9.93 \times 10^{-3}$ & $1.23 \times 10^{-5}$ \\
& & $5 / 2^{+}$ & 1.460 & 0.90 & $6.16 \times 10^{-4}$ \\
\hline \hline
\end{tabular}

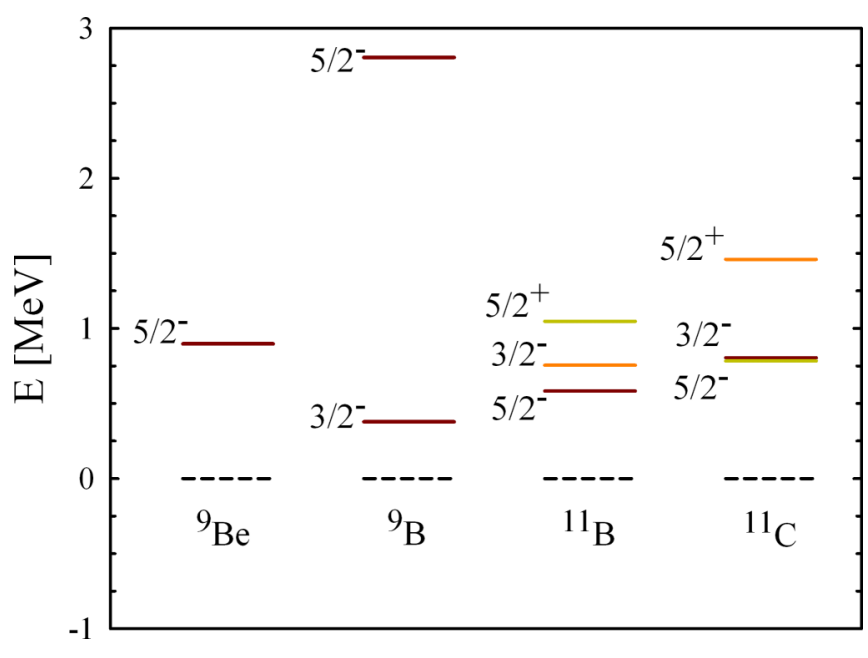
${ }^{11} \mathrm{C}$.

FIG. 11. Spectrum of the Hoyle-analog states in ${ }^{9} \mathrm{Be},{ }^{9} \mathrm{~B},{ }^{11} \mathrm{~B}$, and

a collision of two $\alpha$ particles and a nucleus $X$ ( $X=$ neutron, proton, $\left.{ }^{3} \mathrm{H},{ }^{3} \mathrm{He}\right)$. These resonance states have very small width. We discussed an alternative way for the synthesis of light nuclei in a triple collision, in the same manner as was suggest by Hoyle for ${ }^{12} \mathrm{C}$. We found several resonance states having a total width of a few eV. Most of the obtained resonance states have a width of a few dozens of $\mathrm{keV}$.

In Table X we collect the parameters of the Hoyle-analog states in the light nuclei under consideration. Results presented in this table allow us to formulate the criteria for selecting the Hoyle-analog states. A three-cluster resonance state can be treated as the Hoyle-analog state if the ratio $\Gamma / E<2 \times 10^{-3}$ for this resonance state.

Figure 11 visualizes the results presented in Table $\mathrm{X}$. This figure explicitly demonstrates effects of the Coulomb interaction on the energy of three-cluster resonance states in mirror nuclei ${ }^{9} \mathrm{Be},{ }^{9} \mathrm{~B}$ and ${ }^{11} \mathrm{~B},{ }^{11} \mathrm{C}$. One can see that the Coulomb interaction has a stronger impact on the position of the $5 / 2^{-}$resonance states in ${ }^{9} \mathrm{Be}$ and ${ }^{9} \mathrm{~B}$ than on the position of the $3 / 2^{-}, 5 / 2^{-}$, and $5 / 2^{+}$resonance states in ${ }^{11} \mathrm{~B}$ and ${ }^{11} \mathrm{C}$.

In Table XI we collect all resonance states for the total momentum $J$ and positive parity, where the zero value of the total orbital momentum $(L=0)$ is dominant. This is the case for ${ }^{9} \mathrm{Be},{ }^{9} \mathrm{~B},{ }^{11} \mathrm{~B}$, and ${ }^{11} \mathrm{C}$. The continuous spectrum states with $L=0$ can be interpreted as a head-on collision of the third cluster with the ${ }^{8} \mathrm{Be}$ nucleus being in the $0^{+}$state. As one

TABLE XI. The energy and width of resonance states created by the three-cluster configuration with the total orbital momentum $L=0$.

\begin{tabular}{lccr}
\hline \hline Nucleus & $J^{\pi}$ & $E(\mathrm{MeV})$ & $\Gamma(\mathrm{keV})$ \\
\hline${ }^{9} \mathrm{Be}$ & $1 / 2^{+}$ & 0.338 & 168 \\
${ }^{9} \mathrm{~B}$ & $1 / 2^{+}$ & 0.636 & 477 \\
${ }^{10} \mathrm{~B}$ & $1^{+}$ & 0.604 & 232 \\
${ }^{11} \mathrm{~B}$ & $1 / 2^{+}$ & 0.437 & 15 \\
${ }^{11} \mathrm{C}$ & $1 / 2^{+}$ & 0.906 & 163 \\
\hline \hline
\end{tabular}




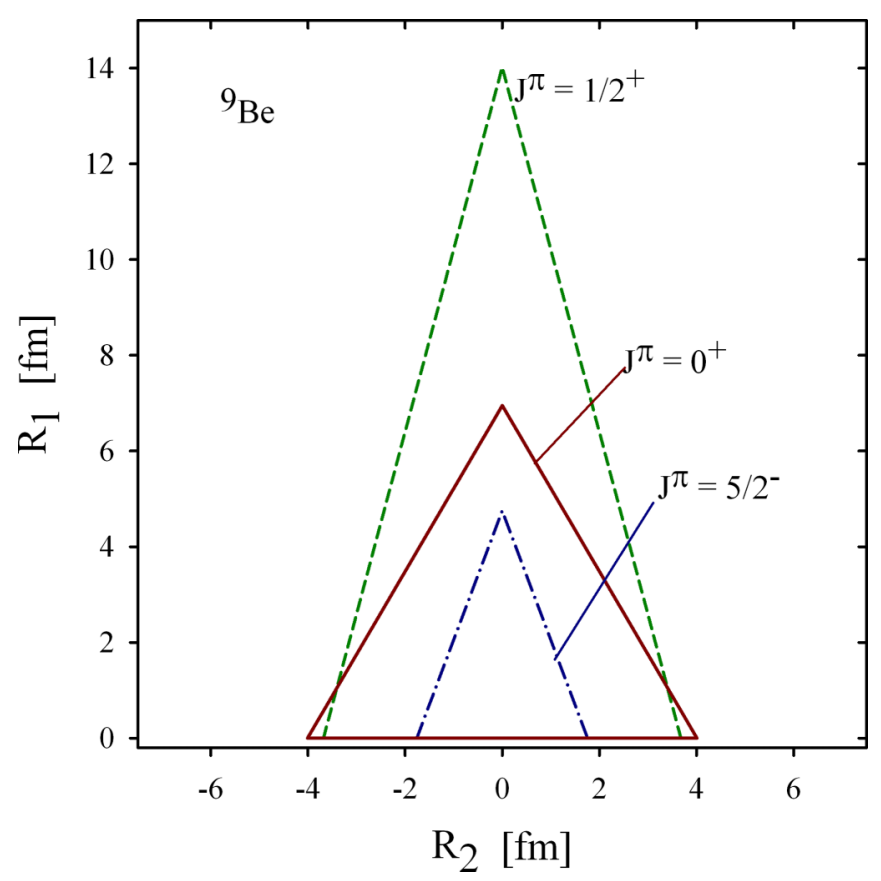

FIG. 12. Shape of triangles for some selected resonance states in ${ }^{9} \mathrm{Be}$ and the Hoyle state $\left(J^{\pi}=0^{+}\right)$.

can see, all these resonance states lie rather close to the threecluster threshold and they are fairly wide, as the total widths are $\Gamma=15 \mathrm{keV}$ or more. Therefor they cannot be considered as the Hoyle-analog states.

Figures 12, 13, and 14 of average distances $R_{1}$ and $R_{2}$ demonstrate the most probable shapes of triangles of threecluster resonance states in ${ }^{9} \mathrm{Be},{ }^{11} \mathrm{~B}$, and ${ }^{11} \mathrm{C}$, respectively.

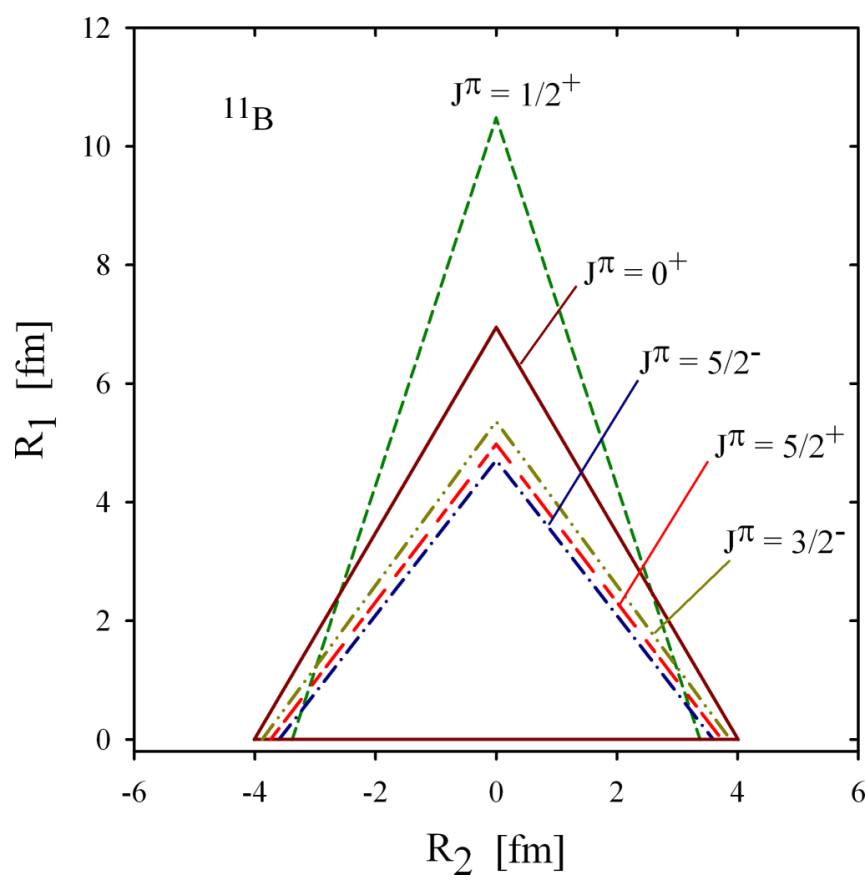

FIG. 13. Shape of triangles of some resonance states in ${ }^{11} \mathrm{~B}$ and the Hoyle resonance state $\left(J^{\pi}=0^{+}\right)$.

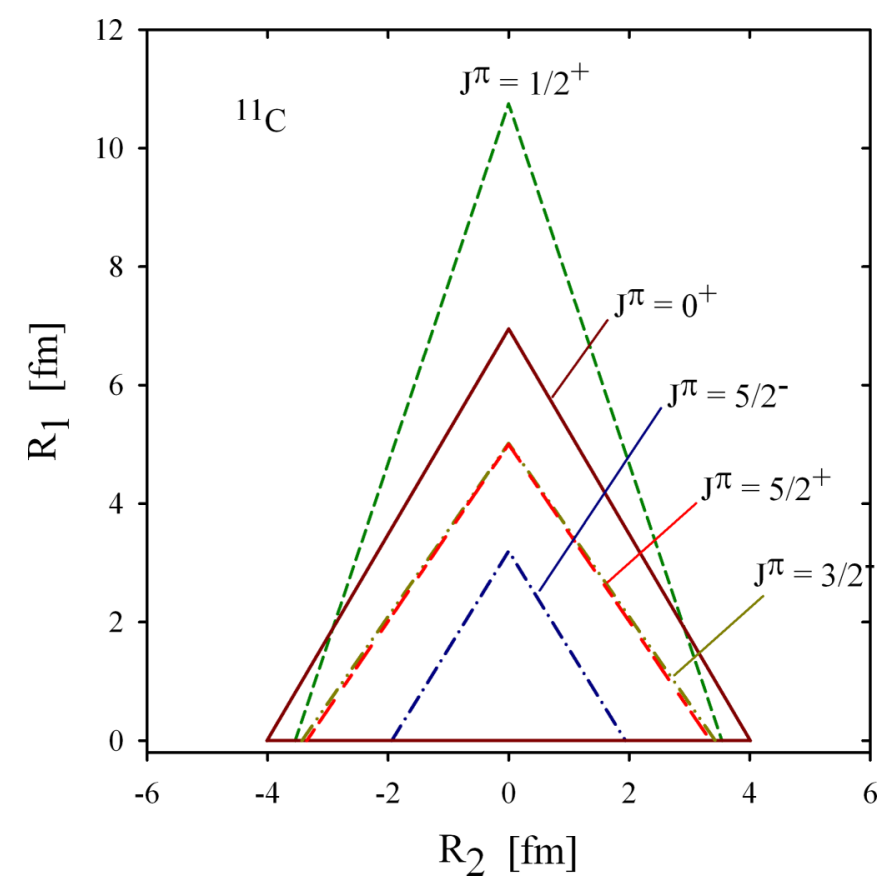

FIG. 14. Shape of triangles of some resonance states in ${ }^{11} \mathrm{C}$ compared to the Hoyle resonance state $\left(J^{\pi}=0^{+}\right)$.

In all these figures we also show the triangle composed of three $\alpha$ particles in the Hoyle resonance state in ${ }^{12} \mathrm{C}$. The $1 / 2^{+}$ resonance states in ${ }^{9} \mathrm{~B},{ }^{11} \mathrm{~B}$, and ${ }^{11} \mathrm{C}$ have very large triangles where a neutron, triton, and ${ }^{3} \mathrm{He}$ nucleus are far away from two $\alpha$ particles. The Hoyle-analog states in these nuclei have a triangle comparable with the shape of the Hoyle state and in some cases (for example, for $J^{\pi}=5 / 2^{-}$) they are more compact.

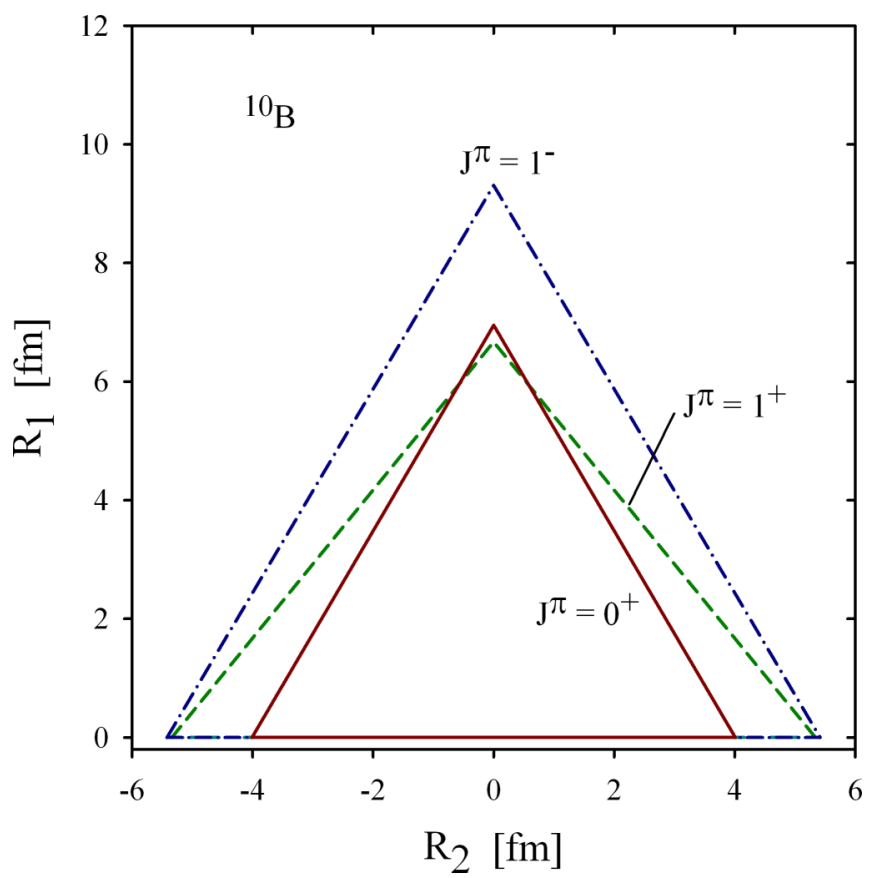

FIG. 15. The most probable shape of three-cluster triangles for most narrow resonance states in ${ }^{10} \mathrm{~B}$ and compared with the Hoyle state $\left(J^{\pi}=0^{+}\right)$. 
Figure 15 demonstrates the shape of triangles for resonance states in ${ }^{10} \mathrm{~B}$. They are the narrowest resonance states; however, the amplitudes of $W_{\mathrm{sh}}$, as shown above, are fairly small and the ratios $\Gamma / E$ for these states are large. They do not match our criteria for the Hoyle-analog states. As we see, the distance between $\alpha$ particles is greater than this distance in the $0^{+}$ resonance state in ${ }^{12} \mathrm{C}$ and all other nuclei considered in the present paper. To this end, the $1 / 2^{+}$resonance states in ${ }^{9} \mathrm{Be},{ }^{9} \mathrm{~B}$, ${ }^{11} \mathrm{~B}$, and ${ }^{11} \mathrm{C}$, which are considered as candidates for the Hoyle states and did not match our criteria, have an average distance between two $\alpha$ particles comparable with the Hoyle state; meanwhile the average distance of the third cluster (neutron, proton, triton, and ${ }^{3} \mathrm{He}$, respectively) to the center of mass of two $\alpha$ particles is very large.

\section{ACKNOWLEDGMENTS}

This work was supported in part by the Program of Fundamental Research of the Physics and Astronomy Department of the National Academy of Sciences of Ukraine (Project No. 0117U000239), by the Ministry of Education and Science of the Republic of Kazakhstan, Research Grant No. IRN: AP 05132476 and by JSPS KAKENHI Grant No. 17K05430.
[1] F. Hoyle, Astrophys. J. Suppl. 1, 121 (1954).

[2] C. W. Cook, W. A. Fowler, C. C. Lauritsen, and T. Lauritsen, Phys. Rev. 107, 508 (1957).

[3] J. H. Kelley, J. E. Purcell, and C. G. Sheu, Nucl. Phys. A 968 , 71 (2017).

[4] M. Freer and H. O. U. Fynbo, Prog. Part. Nucl. Phys. 78, 1 (2014).

[5] H. A. Bethe, Phys. Rev. 55, 434 (1939).

[6] Y. Kanada-En'yo, Phys. Rev. C 75, 024302 (2007).

[7] T. Yamada and Y. Funaki, Phys. Rev. C 82, 064315 (2010).

[8] Y. Kanada-En'yo, T. Suhara, and F. Kobayashi, J. Phys. Conf. Ser. 321, 012009 (2011).

[9] T. Suhara and Y. Kanada-En'yo, Phys. Rev. C 85, 054320 (2012).

[10] T. Yamada and Y. Funaki, Progr. Theor. Phys. Suppl. 196, 388 (2012).

[11] Y. Kanada-En'yo and T. Suhara, Phys. Rev. C 91, 014316 (2015).

[12] B. Zhou and M. Kimura, arXiv:1711.04439.

[13] Y. Chiba and M. Kimura, arXiv:1801.00562.

[14] V. Vasilevsky, A. V. Nesterov, F. Arickx, and J. Broeckhove, Phys. Rev. C 63, 034606 (2001).

[15] V. Vasilevsky, F. Arickx, W. Vanroose, and J. Broeckhove, Phys. Rev. C 85, 034318 (2012).

[16] V. S. Vasilevsky, K. Katō, and N. Z. Takibayev, Phys. Rev. C 96, 034322 (2017).

[17] V. S. Vasilevsky, Ukr. J. Phys. 58, 544 (2013).

[18] A. V. Nesterov, V. S. Vasilevsky, and T. P. Kovalenko, Ukr. J. Phys. 59, 1065 (2014).

[19] V. Vasilevsky, A. V. Nesterov, F. Arickx, and J. Broeckhove, Phys. Rev. C 63, 034607 (2001).

[20] J. Broeckhove, F. Arickx, P. Hellinckx, V. S. Vasilevsky, and A. V. Nesterov, J. Phys. G Nucl. Phys. 34, 1955 (2007).
[21] A. V. Nesterov, F. Arickx, J. Broeckhove, and V. S. Vasilevsky, Phys. Part. Nucl. 41, 716 (2010).

[22] A. V. Nesterov, V. S. Vasilevsky, and T. P. Kovalenko, Phys. At. Nucl. 77, 555 (2014).

[23] W. Zickendraht, Ann. Phys. (NY) 35, 18 (1965).

[24] A. Avery, Hyperspherical Harmonics. Applications in Quantum Theory (Kluwer, Dordrecht, 1989).

[25] R. I. Dzhibuti and N. B. Krupennikova, The Method of Hyperspherical Functions in the Quantum Mechanics of Several Bodies (in Russian) (Metsniereba, Tbilisi, 1984).

[26] F.Zernike and H. C. Brinkman, Proc. Kon. Ned. Acad. Wetensch. Amsterdam 38, 161 (1935).

[27] M. Abramowitz and A. Stegun, Handbook of Mathematical Functions (Dover, New-York, 1972).

[28] Y. C. Tang, M. Lemere, and D. R. Thompsom, Phys. Rep. 47, 167 (1978).

[29] V. S. Vasilevsky, Y. A. Lashko, and G. F. Filippov, Phys. Rev. C 97, 064605 (2018).

[30] G. F. Filippov and I. P. Okhrimenko, Yad. Fiz. 32, 932 (1980) [Sov. J. Nucl. Phys. 32, 480 (1980)].

[31] G. F. Filippov, Yad. Fiz. 33, 928 (1981) [Sov. J. Nucl. Phys. 33, 488 (1981)].

[32] D. R. Thompson, M. LeMere, and Y. C. Tang, Nucl. Phys. A 286, 53 (1977).

[33] I. Reichstein and Y. C. Tang, Nucl. Phys. A 158, 529 (1970).

[34] A. Hasegawa and S. Nagata, Prog. Theor. Phys. 45, 1786 (1971).

[35] F. Tanabe, A. Tohsaki, and R. Tamagaki, Prog. Theor. Phys. 53, 677 (1975)

[36] C. Kurokawa and K. Katō, Nucl. Phys. A 792, 87 (2007).

[37] T. Yoshida, N. Itagaki, and K. Katō, Phys. Rev. C 83, 024301 (2011).

[38] T. Neff and H. Feldmeier, Few-Body Syst. 45, 145 (2009). 